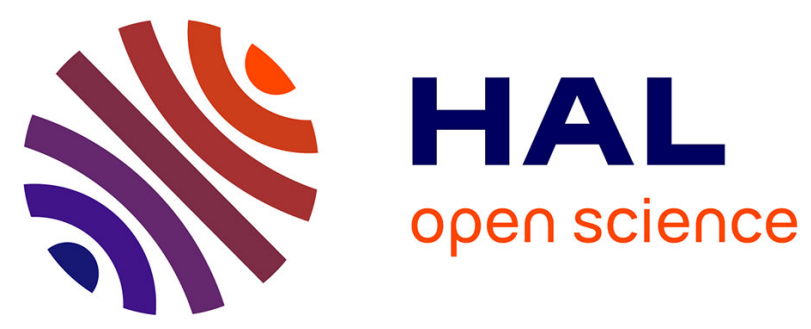

\title{
REDUCTION TYPES OF GENUS-3 CURVES IN A SPECIAL STRATUM OF THEIR MODULI SPACE
}

Irene Bouw, Nirvana Coppola, Pinar Kilicer, Sabrina Kunzweiler, Elisa Lorenzo García, Anna Somoza

\section{> To cite this version:}

Irene Bouw, Nirvana Coppola, Pinar Kilicer, Sabrina Kunzweiler, Elisa Lorenzo García, et al.. REDUCTION TYPES OF GENUS-3 CURVES IN A SPECIAL STRATUM OF THEIR MODULI SPACE. 2020. hal-02512861

\section{HAL Id: hal-02512861 \\ https://hal.science/hal-02512861}

Preprint submitted on 20 Mar 2020

HAL is a multi-disciplinary open access archive for the deposit and dissemination of scientific research documents, whether they are published or not. The documents may come from teaching and research institutions in France or abroad, or from public or private research centers.
L'archive ouverte pluridisciplinaire HAL, est destinée au dépôt et à la diffusion de documents scientifiques de niveau recherche, publiés ou non, émanant des établissements d'enseignement et de recherche français ou étrangers, des laboratoires publics ou privés. 


\title{
REDUCTION TYPES OF GENUS-3 CURVES IN A SPECIAL STRATUM OF THEIR MODULI SPACE
}

\author{
IRENE BOUW, NIRVANA COPPOLA, PINAR KILIÇER, SABRINA KUNZWEILER, \\ ELISA LORENZO GARCÍA, AND ANNA SOMOZA
}

\begin{abstract}
We study a 3 -dimensional stratum $\mathcal{M}_{3, V}$ of the moduli space $\mathcal{M}_{3}$ of curves of genus 3 parameterizing curves $Y$ that admit a certain action of $V \simeq C_{2} \times C_{2}$. We determine the possible types of the stable reduction of these curves to characteristic different from 2. We define invariants for $\mathcal{M}_{3, V}$ and characterize the occurrence of each of the reduction types in terms of them. We also calculate the $j$-invariant (resp. the Igusa invariants) of the irreducible components of positive genus of the stable reduction $Y$ in terms of the invariants.
\end{abstract}

\section{INTRODUCTION}

Let $(K, \nu)$ be a discrete valuation field, $\mathcal{O}$ its ring of integers, and $k$ its residue field. Let $Y$ be a smooth projective and absolutely irreducible curve over $K$ of genus $g(Y) \geq 1$. A theorem of Deligne-Mumford [DM69] states that after replacing $K$ by a finite extension there exists a semistable model $\mathcal{Y}$ over $\operatorname{Spec}(\mathcal{O})$ (see Definition 2.4). If $g(Y) \geq 2$ there exists a unique minimal semistable model, which we call the stable model. Its special fiber $\bar{Y}$ is called the stable reduction of $Y$. For fixed genus $g \geq 2$ there are only finitely many possibilities for the reduction type, i.e., the graph of irreducible components of $\bar{Y}$ together with the genus of the normalization of each irreducible component. If $\bar{Y}$ is smooth we say that $Y$ has potentially good reduction.

In the case that $g(Y)=1$ a minimal semistable model does not need to be unique, but the reduction type does not depend on the choice of a minimal semistable model. It is determined by the $j$-invariant $j(Y)$ of $Y$. Namely, the special fiber $\bar{Y}$ of any minimal semistable model of $Y$ is smooth if and only if the valuation $\nu(j(Y))$ is non-negative and $\bar{Y}$ is a projective line that intersects itself in one point (multiplicative reduction) otherwise. (See for example [Sil09, Chapter VII, Prop. 5.5]).

In [Liu93, Théorème 1] Liu has generalized this results to curves of genus 2. He determines the reduction types in terms of the Igusa invariants. Moreover, Liu gives an expression for the $j$-invariant of the irreducible components of positive genus in terms of the Igusa invariants in the case that $\bar{Y}$ is not smooth. (If $\bar{Y}$ is singular, then the irreducible components of positive genus are necessarily elliptic curves.)

Smooth projective curves of genus 3 are either hyperelliptic or plane quartics, where the latter form a dense open subset of the moduli space $\mathcal{M}_{3}$ of curves of genus 3 . The Dixmier-Ohno invariants for smooth quartics have similar properties as the Igusa invariants for curves of genus 2 and the $j$-invariant for elliptic curves. The Dixmier-Ohno invariants do not extend to the locus of smooth hyperelliptic curves, and one should consider the locus in $\mathcal{M}_{3}$ of smooth hyperelliptic curves as a part of the boundary of the locus of smooth quartics in the Deligne-Mumford compactification $\overline{\mathcal{M}_{3}}$. There exists a different set of invariants, called the Shioda invariants, for smooth hyperelliptic curves of genus 3 .

2010 Mathematics Subject Classification. 14H10 (primary); 14H50, 14H25, 11G20, 14Q05 (secondary). 
It is natural to ask whether one can characterize the reduction types of smooth plane quartics in terms of the Dixmier-Ohno invariants. The first result in this direction is proved in [LLLR19. The authors of loc. cit. characterize in terms of the DixmierOhno invariants when the stable reduction $\bar{Y}$ of a smooth plane quartic is a smooth hyperelliptic curve. The authors of [LLLR19] also compute the Shioda invariants of the smooth hyperelliptic curve $\bar{Y}$.

An alternative direction to generalize the result of Liu is to consider families of superelliptic curves, which are cyclic covers $f: Y \rightarrow \mathbb{P}_{K}^{1}$ of the projective line. In the case that either $\operatorname{char}(k) \nmid \operatorname{deg}(f)$ or $\operatorname{char}(k)=\operatorname{deg}(f)=p$ there exist algorithms to compute the stable reduction $\bar{Y}$ (see for example [BW17] for the general case or [DDMM19] for hyperelliptic curves). However, this approach does not yield a natural interpretation in terms of the Dixmier-Ohno invariants. An interesting special case is the case of Picard curves, which are both plane quartics and superelliptic curves. In this case the possible reduction types can be found in [BBW17] and [BKSW]. The case of Picard curves with potentially good reduction in terms of the Dixmier-Ohno invariants is described in [LLLR19, Section 4.2].

The motivating question for the current paper is whether it is possible to characterize the reduction type of a smooth plane quartic in terms of the Dixmier-Ohno invariants also in the case that the stable reduction $\bar{Y}$ is not smooth. Due to the many different reduction types we restrict to a specific 3 -dimensional stratum in $\mathcal{M}_{3}$. In this paper we determine all possible reduction types for this stratum and prove a result analogous to Liu's result for curves of genus 2 .

We now describe our results in more detail. In the rest of the introduction we assume that both $K$ and $k$ have characteristic different from 2 . We consider the stratum $\mathcal{M}_{3, V} \subset$ $\mathcal{M}_{3}$ consisting of smooth curves $Y / K$ of genus 3 such that $\operatorname{Aut}_{\bar{K}}(Y)$ contains a subgroup $V \simeq C_{2} \times C_{2}$ with $g(Y / V)=0$ and such that all degree-2 subcovers of $Y \rightarrow X:=Y / V$ have genus 1 .

The study of these curves goes back to Ciani in 1899 ([Cia99]). They are sometimes called Ciani surfaces in his honor. More recent references are [LR08] and [HLP00]. In [HLP00] the authors find curves from this family with many rational points and small conductor (see also Example 3.10).

A dense open set of $\mathcal{M}_{3, V}$ parametrizes smooth plane quartics with an action of the Klein 4-group $V$. Over a sufficiently large field, these curves may be described by an explicit quartic equation in standard form (see Lemma 2.2), and one can express the Dixmier-Ohno invariants in terms of the coefficients of this equation. In Section 3.1 we replace the full set of Dixmier-Ohno invariants by a smaller set $I_{3}, I_{3}^{\prime}, I_{3}^{\prime \prime}, I_{6}$ of invariants, which are easier to handle in our set-up. They may be expressed in terms of the DixmierOhno invariants (see $\left[\mathrm{BCK}^{+} 20\right]$ ). Our main results are formulated in terms of these invariants in Section 3.2 .

All curves $Y$ parametrized by $\mathcal{M}_{3, V}$ admit a $V$-Galois cover $f: Y \rightarrow \mathbb{P}^{1}$. This allows us to calculate the possible reduction types by extending the method of [BW17] to our situation. The key ideas of this method in our set-up are explained in Sections 2.2 2.4.

Combining these two approaches, we show in Theorem 2.11 that there are exactly 13 reduction types for curves parametrized by $\mathcal{M}_{3, V}$. The different types are illustrated in Appendix A. Proposition 3.6 characterizes potentially good quartic reduction. The analogous result in the case of potentially good hyperelliptic reduction can be found in Proposition 5.5. In Theorems 3.7 and 3.8 we give explicit conditions in terms of the invariants $I_{3}, I_{3}^{\prime}, I_{3}^{\prime \prime}, I_{6}$ characterizing the different reduction types. Moreover, we 
determine the Igusa invariants (respectively the $j$-invariant) of the irreducible components of positive genus in the case that $\bar{Y}$ is singular (see the proofs in Section 4). In Section 5 we discuss the case where the curve $Y$ is hyperelliptic. We prove the analogous result in Theorem 5.6, which is phrased in terms of a modified set of invariants described in Section 5.1.

Acknowlegments. This project began at the Women in Numbers Europe 3 workshop in Rennes, August 2019. We are grateful to the organizers for bringing us together and providing us with an excellent working environment to get this project underway. We thank Christophe Ritzenthaler for his ideas for the proofs of Propositions 3.4, 3.6, 5.2 and 5.5 .

\subsection{Notation.}

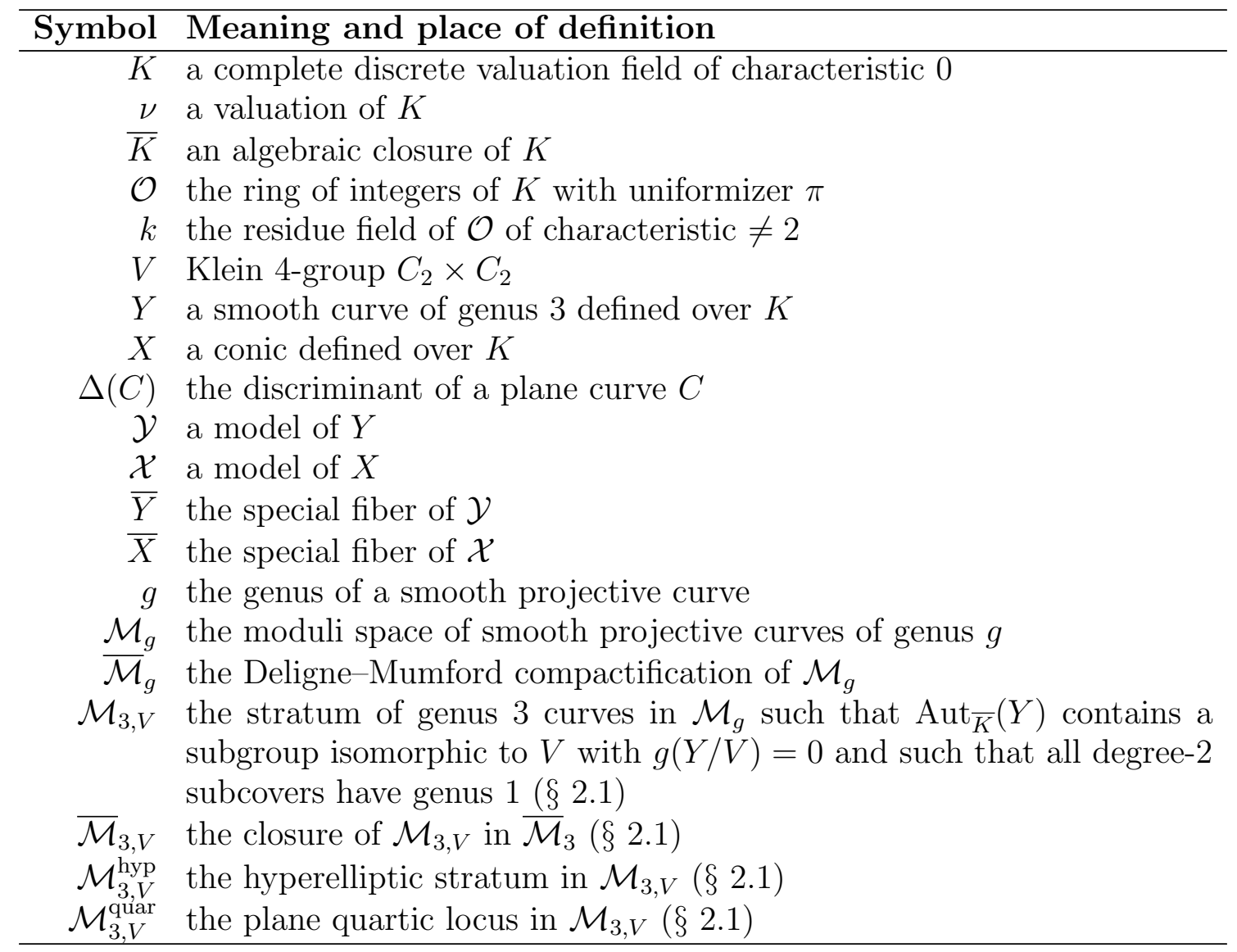

\section{Stable REDUCtion AND ADMissible COVERS}

2.1. The set-up. Let $\bar{K}$ be an algebraically closed field of characteristic $2 \neq p \geq 0$. We denote by $\mathcal{M}_{g}$ the moduli space of smooth projective curves of genus $g$ defined over $K$. In this paper we consider the locus $\mathcal{M}_{3, V}$ of curves of genus 3 such that $\operatorname{Aut}_{\bar{K}}(Y)$ contains a subgroup isomorphic to the Klein 4-group $V \simeq C_{2} \times C_{2}$ such that $X:=Y / V$ has genus 0 and each of the intermediate covers of degree 2 has genus 1 . Since we assume $\operatorname{char}(K) \neq 2$, the cover $f: Y \rightarrow X$ is tamely ramified. The following lemma states some elementary facts on such covers.

Lemma 2.1. Let $Y / \bar{K}$ be a smooth projective curve of genus 3 , and $f: Y \rightarrow X \simeq \mathbb{P} \frac{1}{K}$ a Galois cover with Galois group isomorphic to $V$ such that all intermediate covers of degree 2 have genus 1. 
(1) Every element of order 2 in $V$ generates the inertia group of exactly 2 branch points of $f$.

(2) If $Y$ is hyperelliptic, then $\operatorname{Aut}_{\bar{K}}(Y)$ contains a subgroup $A \simeq\left(C_{2}\right)^{3}$. The hyperelliptic involution $\iota$ acts on $X$ by interchanging the two branch points of $f$ with the same inertia generator. The cover $Y \rightarrow Y / A$ of degree 8 is branched at 5 points. Two of these have inertia generator $\iota$, the other three one of the elements of order 2 contained in a unique subgroup $V \subset A$.

(3) The stratum $\mathcal{M}_{3, V}$ is irreducible and of dimension 3. The hyperelliptic curves form an irreducible substratum $\mathcal{M}_{3, V}^{\text {hyp }}$ of dimension 2.

Proof. Statement (1) follows from the Riemann-Hurwitz formula applied to $f$ and each of its subcovers of degree 2. Statement (2) follows similarly by considering the cover $Y \rightarrow Y / A$ and its subcovers. Statement (3) is well-known, see e.g. [LRRS14]. It also may be deduced from (1) and (2).

We denote by $\mathcal{M}_{3, V}^{\text {quar }}=\mathcal{M}_{3, V} \backslash \mathcal{M}_{3, V}^{\text {hyp }}$ for the locus of smooth plane quartics with $V \subset \operatorname{Aut}_{\bar{K}}(Y)$. We write $\overline{\mathcal{M}}_{3}$ for the Deligne-Mumford compactification of $\mathcal{M}_{3}$ and $\overline{\mathcal{M}}_{3, V}$ for the closure of $\mathcal{M}_{3, V}$ in $\overline{\mathcal{M}}_{3}$. A $\bar{K}$-point of the boundary of $\overline{\mathcal{M}}_{3, V}$ consists of a stable curve $\bar{Y}$ of genus 3 on which $V$ acts faithfully with a quotient of genus zero. The goal of this paper is to study the "types" of stable curves occurring in the boundary of $\overline{\mathcal{M}}_{3, V}$. Rather than working out necessary and sufficient conditions for a $V$-action on a stable curve $\bar{Y}$ of genus 3 to correspond to a point of this boundary, we determine instead the compactification $\overline{\mathcal{H}}_{3, V}$ of the Hurwitz space parametrizing $V$-Galois covers $Y \rightarrow X \simeq \mathbb{P}_{\bar{K}}^{1}$ as in Lemma 2.1. We refer to [RW06] for precise definitions and properties of Hurwitz spaces. There are different variants, but for our purposes it is not necessary to specify which one we use.

Lemma 2.1.(3) implies that to determine the types of stable curves occurring in the boundary of $\overline{\mathcal{M}}_{3, V}$ it suffices to consider the possible degenerations of the non-hyperelliptic curves of genus 3, i.e., smooth plane quartics.

The non-hyperelliptic curves of genus 3 with non-trivial automorphism group (over $\mathbb{C}$ ) have been classified by Vermeulen [Ver83] (see also [Hen76]). In [LRRS14 it is shown that this classification also holds in positive characteristic with a few exceptional cases in small characteristic. The following result is a special case of this classification. Actually, one may additionally assume that the parameters $A, B, C$ are equal to 1 .

Lemma 2.2. Let $Y / \bar{K}$ be a smooth plane quartic such that there exists a subgroup $V \subseteq$ $\operatorname{Aut}_{\bar{K}}(Y)$.

(1) Then $Y$ may be defined by an equation

$$
Y: F:=A x^{4}+B y^{4}+C z^{4}+a y^{2} z^{2}+b x^{2} z^{2}+c x^{2} y^{2}=0
$$

and the elements of $V$ act as $(x: y: z) \mapsto( \pm x: \pm y: z)$.

(2) The ramification points of $f: Y \rightarrow Y / V=: X$ are the points with $x y z=0$.

Proof. Statement (1) follows from the classification of non-hyperelliptic curves of genus 3 with non-trivial automorphism group ([LRRS14]). Note that for a plane quartic $Y$ the existence of a subgroup of $\operatorname{Aut}_{\bar{K}}(Y)$ isomorphic to $V$ already implies that $g(Y / V)=0$ and that the degree-2 subcovers have genus 1. Statement (2) follows by direct verification.

In the rest of the paper, if $Y$ is a smooth plane quartic, then we denote the elements of $V$ as

$\sigma_{a}((x: y: z))=(-x: y: z), \quad \sigma_{b}((x: y: z))=(x:-y: z), \quad \sigma_{c}((x: y: z))=(x: y:-z)$. 
We find that $X:=Y / V$ admits an equation of the form

$$
X: A u^{2}+B v^{2}+C w^{2}+a v w+b u w+c u v=0,
$$

where $u=x^{2}, v=y^{2}$, and $w=z^{2}$ and the map $f: Y \rightarrow X$ is given by $(x: y: z) \mapsto(u$ : $v: w)$.

For $i \in\{a, b, c\}$ we define $E_{i}=Y /\left\langle\sigma_{i}\right\rangle$. Then $E_{i}$ is an elliptic curve for all $i$, see Lemma 2.2. We obtain the following diagram.

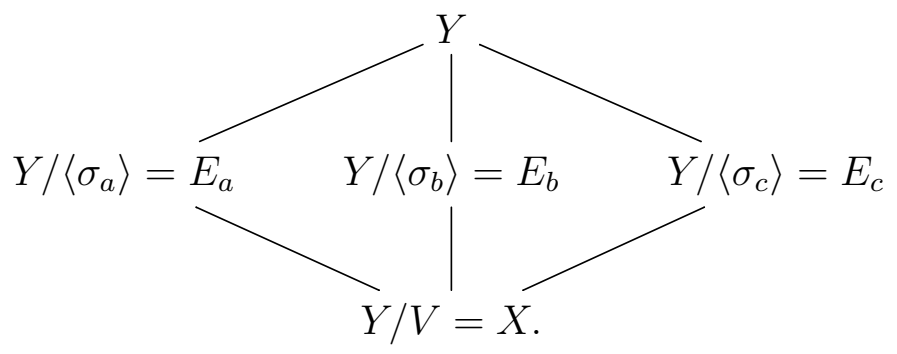

Set

(2.3) $p_{a}(T)=T^{2}-2 a T+4 B C, \quad p_{b}(T)=T^{2}-2 b T+4 A C, \quad p_{c}(T)=T^{2}-2 c T+4 A B$.

For $i \in\{a, b, c\}$ we let $4 \Delta_{i}$ be the discriminant of $p_{i}$, i.e.,

$$
\Delta_{a}=a^{2}-4 B C, \quad \Delta_{b}=b^{2}-4 A C, \quad \Delta_{c}=c^{2}-4 A B .
$$

One computes

$$
\begin{aligned}
& \Delta(X)=-4 A B C+A a^{2}+B b^{2}+C c^{2}-a b c, \\
& \Delta(Y)=-2^{-20} A B C \Delta_{a}^{2} \Delta_{b}^{2} \Delta_{c}^{2} \Delta(X)^{4},
\end{aligned}
$$

Here $\Delta(X)$ and $\Delta(Y)$ are the discriminants of $X$ and $Y$ as hypersurfaces (see for example [Dem12]), respectively.

Remark 2.3. The Jacobian of $Y$ is isogenous to $E_{a} \times E_{b} \times E_{c}$. In [HLP00, Prop. 15] the isomorphism class of the Jacobian over an explicit finite extension of $K$ is determined. The authors of that paper use this to find an example of a curve in this family with small conductor ([HLP00, Example 16]).

Let $\alpha$ be a root of $p_{a}, \beta$ a root of $p_{b}$, and $\gamma$ a root of $p_{c}$. Then the branch points of the cover $f: Y \rightarrow X$ are

$$
\begin{array}{ll}
\sigma_{a}: P_{a}=(0: \alpha:-2 B), & P_{a}^{\prime}=(0:-2 C: \alpha), \\
\sigma_{b}: P_{b}=(-2 C: 0: \beta), & P_{b}^{\prime}=(\beta: 0:-2 A), \\
\sigma_{c}: P_{c}=(\gamma:-2 A: 0), & P_{c}^{\prime}=(-2 B: \gamma: 0) .
\end{array}
$$

The group element $\sigma_{i}$ is the inertia generator of the points $P_{i}$ and $P_{i}^{\prime}$ for $i \in\{a, b, c\}$.

2.2. Stable reduction of covers. In this section $(K, \nu)$ is a complete discrete valuation field. We assume that the characteristic of $K$ is 0 to avoid some subtleties in small characteristic. However, we can extend the results to the case of characteristic $p \neq 2$. We denote by $\mathcal{O}$ the ring of integers of $K$, by $\pi$ a uniformizing element, and by $k$ its residue field. We assume that $k$ is algebraically closed and of characteristic $\neq 2$. We allow $K$ to be replaced by a finite extension. We assume the valuation to be normalized by $\nu(p)=1$, where $p>0$ is the residue characteristic of $\nu$. In the case of equal characteristic 0 , one adapts this choice of normalization suitably. 
Let $Y$ be a smooth projective absolutely irreducible curve over $K$. A model of $Y$ is a flat proper normal $\mathcal{O}$-scheme $\mathcal{Y}$ such that $\mathcal{Y} \otimes_{\mathcal{O}} K \simeq Y$. If the model is clear from the context, we call its special fiber the reduction of $Y$ and denote it by $\bar{Y}$.

Definition 2.4. (1) A curve $Y$ over $K$ has good reduction if there exists a model of $Y$ over $\mathcal{O}$ such that $\bar{Y}$ is smooth.

(2) A curve $Y$ over $K$ has potentially good reduction if it has good reduction after replacing $K$ by a finite extension.

(3) A curve $Y$ over $K$ has geometric bad reduction if it does not have potentially good reduction.

(4) A curve $Y$ over $K$ has semistable reduction if there exists a model $\mathcal{Y}$ of $Y$ whose special fiber $\bar{Y}$ is semistable, i.e., is reduced and has at most ordinary double points as singularities.

The Stable Reduction Theorem of Deligne-Mumford [DM69] states that every curve $Y$ of genus $g(Y) \geq 2$ admits a semistable model after replacing $K$ by a finite extension, if necessary. Moreover, there exists a unique minimal semistable model $\mathcal{Y}^{\text {st }}$, which is called the stable model of $Y$. Its special fiber $\bar{Y}$ is characterized by the property that each irreducible component of genus 0 intersects the rest of $\bar{Y}$ in at least three points. Every semistable model $\mathcal{Y}$ admits a surjective map $\mathcal{Y} \rightarrow \mathcal{Y}^{\text {st }}$, which contracts the "superfluous" irreducible components of genus 0 of the special fiber and is an isomorphism on the generic fiber.

After replacing $K$ by a finite extension, we may assume that the branch points of $f$ are $K$-rational. Let $D \subset X$ be the branch locus of $f$. We consider it as marking on $X$. A semistable $\mathcal{O}$-model $(\mathcal{X}, \mathcal{D})$ of the marked curve $(X, D)$ is a semistable model $\mathcal{X}$ of $X$ over $\mathcal{O}$, together with a relative divisor $\mathcal{D} \rightarrow \operatorname{Spec}(\mathcal{O})$ with $D=\mathcal{D} \otimes_{\mathcal{O}} K$. Here $\mathcal{D}$ is the union of disjoint sections $s_{1}, \ldots, s_{r}: \operatorname{Spec}(\mathcal{O}) \rightarrow \mathcal{X}^{\mathrm{sm}}$ into the smooth locus of $\mathcal{X}$.

The following result is a version of the Stable Reduction Theorem for covers. It follows immediately from [BW17, Prop. 3.2]. Note that the conditions of the following result are satisfied in our situation.

Proposition 2.5. Let $f: Y \rightarrow X \simeq \mathbb{P}_{K}^{1}$ be a $G$-Galois cover over $K$ such that the branch points of $f$ are $K$-rational, the number of branch points is greater than or equal to 3 , and the residue characteristic of $K$ does not divide the order of $G$.

(1) There exists a unique minimal semistable model $(\mathcal{X}, \mathcal{D})$ of the marked curve $(X, D)$.

(2) The special fiber $\bar{X}$ of $\mathcal{X}$ is a tree of projective lines. Every irreducible component $\bar{X}_{i}$ of $\bar{X}$ contains at least three points which are either singular points of $\bar{X}$ or belong to the support of $\bar{D}$.

We call the model $(\mathcal{X}, \mathcal{D})$ from Proposition 2.5 the stably marked model of $(X, D)$. The normalization $\mathcal{Y}$ of $\mathcal{X}$ in the function field $K(\bar{Y})$ of $Y$ is a model of $Y$. After replacing $K$ by a further finite extension, we may assume that the special fiber $\bar{Y}$ of $\mathcal{Y}$ is reduced.

Proposition 2.6. (1) The model $\mathcal{Y}$ of $Y$ is semistable.

(2) The map $f$ extends to a finite flat map $f: \mathcal{Y} \rightarrow \mathcal{X}$ over $\mathcal{O}$.

(3) The special fiber $\bar{f}: \bar{Y} \rightarrow \bar{X}$ of $f: \mathcal{Y} \rightarrow \mathcal{X}$ is an admissible cover (Def. 2. 7).

(4) Every admissible $G$-Galois cover $\bar{f}: \bar{Y} \rightarrow \bar{X}$ over $k$ occurs via this construction.

Proof. Statements (1) and (2) follow from [BW17, Theorem 3.4]. Statements (3) and (4) are proved in [Wew99]. Here we use that the residue characteristic of $K$ does not divide the cardinality of the Galois group. 
The special fiber $\bar{f}: \bar{Y} \rightarrow \bar{X}$ of the map $f: \mathcal{Y} \rightarrow \mathcal{X}$ from Proposition 2.6.(2) is called the stable reduction of $f$.

Definition 2.7. An admissible cover is a G-Galois cover $\bar{f}: \bar{Y} \rightarrow \bar{X}$ between projective semistable irreducible curves over an algebraically closed field such that:

(1) The singular points of $\bar{Y}$ map to the singular points of $\bar{X}$.

(2) For every singular point $\tau \in \bar{Y}$ the inertia group of $\tau$ acts on the two branches of $\bar{Y}$ via characters that are inverse to each other.

We refer to [RW06, Section 5] or [Wew99] for more details on admissible covers and their deformation. In our situation all elements of the Galois group $V$ have order 1 or 2, and condition (2) of Definition 2.7 reduces to the condition that the inertia group of the restriction of $\bar{f}$ to each of the branches of $\bar{Y}$ at the singular point $\tau$ is the same subgroup of $V$.

2.3. Combinatorial description of the stable reduction. The boundary of the Hurwitz space $\overline{\mathcal{H}}_{3, V}$ parametrizes admissible $V$-Galois covers (Definition 2.7). The natural map

$$
\overline{\mathcal{H}}_{3, V} \rightarrow \overline{\mathcal{M}}_{3, V}, \quad[Y \rightarrow X] \mapsto Y^{\mathrm{st}},
$$

is finite and surjective. Here $Y^{\text {st }}$ is the stable curve of genus 3 obtained by contracting all irreducible components of genus 0 that intersect the rest of $\bar{Y}$ in at most two points. To find all possibilities for the stable curves in the boundary of $\overline{\mathcal{M}}_{3, V}$ it therefore suffices to find all possibilities for the corresponding admissible covers by Proposition 2.6.(4). In this section we give a combinatorial description for the admissible $V$-Galois covers arising as the reduction of the Galois covers described in Lemma 2.1. For simplicity, we call these covers simply admissible $V$-Galois covers.

Definition 2.8. A decorated graph over $k$ is a datum $(\bar{X}, \bar{D})$, where

(1) $\bar{X}$ is a semistable curve of genus 0 over $k$, i.e., a tree of projective lines,

(2) $\bar{D} \subset \bar{X}^{\text {sm }}$ is a set of smooth $k$-rational points of cardinality 6 ,

(3) every irreducible component of $\bar{X}$ contains at least three points that are either in $\bar{D}$ or singular points of $\bar{X}$,

(4) the points of $\bar{D}$ are labelled by one of $\{1,2,3\}$, where each label occurs exactly twice.

Definition 2.8 may easily be adapted to a more general set-up. In this paper the notion always refers to our particular set-up.

The following lemma follows from the fact that $\bar{X}$ is a tree, together with the description of the fundamental group of an affine curve of genus 0 .

Lemma 2.9. Let $(\bar{X}, \bar{D})$ be a decorated graph. Then there is a unique way to label the singular points of $\bar{X}$ by one of $\{0,1,2,3\}$ such that the product of the inertia generators $\sigma_{i} \in V$ of the marked and singular points on each irreducible component of $\bar{X}$ is the trivial element of $V$.

Let $\bar{f}: \bar{Y} \rightarrow \bar{X}$ be an admissible $V$-Galois cover. Write $\bar{D} \subset \bar{X}^{\text {sm }}$ for the branch points of $\bar{f}$ contained in the smooth locus $\bar{X}^{\mathrm{sm}}$ of $\bar{X}$. Proposition 2.5 and Lemma 2.1 imply that every choice of a numbering of the elements of order 2 of $V$ gives rise to a decorated graph. In fact one checks that the labeling of a singular point $\tau$ of $\bar{X}$ given by Lemma 2.9 is 0 if and only if $\bar{f}$ is unbranched above $\tau$. If the label of $\tau$ is non-zero, it corresponds to the inertia generator of $\tau$ otherwise. This follows from the fact that the restriction of $\bar{f}$ to an irreducible component of $\bar{Y}$ above $\bar{X}_{t}$ is a tamely ramified cover of $\bar{X}_{t} \simeq \mathbb{P}_{k}^{1}$. 
The following lemma is straightforward. A similar statement in the case of cyclic covers can be found in [BW17, Section 4].

Lemma 2.10. Let $(\bar{X}, \bar{D})$ be a decorated graph. Choose a numbering $\left\{\sigma_{1}, \sigma_{2}, \sigma_{3}\right\}$ of the elements of order 2 of $V$. There exists a unique admissible $V$-Galois cover $\bar{f}: \bar{Y} \rightarrow \bar{X}$ such that the inertia generator of $x \in \bar{D}$ is given by its label and $\bar{f}$ is unbranched outside $\bar{D} \cup \bar{X}^{\text {sing }}$. The decorated graphs are listed in Appendix $A$.

In the following theorem we determine the different possibilities for the stable reduction of $Y$ in our situation. By type of a stable curve we mean the intersection graph of its irreducible components together with the genus of each of the irreducible components.

Theorem 2.11. Let $Y / K$ be a smooth projective curve of genus 3 such that there exists a subgroup $V \subseteq \operatorname{Aut}_{\bar{K}}(Y)$ with $g(Y / V)=0$ and such that all subcovers of $Y \rightarrow Y / V$ of degree 2 have genus 1 . Then there are 13 different possibilities for the type of stable reduction of $Y$.

Proof. Let $X=Y / V$ and $D$ be the branch locus of $f: Y \rightarrow X$, considered as a divisor on $X$. After replacing $K$ by a finite extension, we may assume that the divisor $D$ splits over $K$. It follows by Proposition 2.5 that $(X, D)$ admits a stable model $(\mathcal{X}, \mathcal{D})$. Its special fiber $(\bar{X}, \bar{D})$ gives rise to a decorated graph (Definition 2.8).

Since $\bar{D}$ has cardinality 6 and $(\bar{X}, \bar{D})$ must fulfill Condition (iii) from Definition 2.8 , the curve $\bar{X}$ cannot have more than 4 components. There are the following possibilities for $\bar{X}$ :

(I) $\bar{X}$ is irreducible.

(II) $\bar{X}$ is a chain of two projective lines. Either there are three marked points on each component or there are four marked points on one component and two marked points on the other.

(III) $\bar{X}$ is a chain of three projective lines. Either there are exactly two marked points on each component or there is only one marked point on the middle component and two, respectively three, marked points on the remaining components.

(IV) $\bar{X}$ is a chain of four projective lines and there is one marked point on each of the two components in the middle and two marked points on the other two components.

$\left(\mathrm{IV}^{*}\right) \bar{X}$ consists of four projective lines such that the first component intersects all other components and there are no further singularities. There is no marked point on the first component and two marked points on each of the remaining components.

Next, we assign a label in $\{0,1,2,3\}$ to the points in $\bar{D}$ and the set $S$ of singularities of $\bar{X}$. Let $\sigma_{1}, \sigma_{2}, \sigma_{3}$ denote the non-trivial elements of $V$. Lemma 2.10 states that the label on the singularities is uniquely determined by that of the marked points. Moreover, none of the marked points is assigned the label 0 .

Up to the choice of the numbering of the elements of order 2 in $V$, there are 20 possibilities for the decorated graph. These are depicted in Figures $(\mathrm{I})-\left(\overline{\mathrm{IV}^{*}}\right)$ in Appendix A. The numbering of the cases in the rest of the proof refers to the numbers of the cases there.

To finish the proof it remains to describe the admissible cover $\bar{Y} \rightarrow \bar{X}$ and the image of $\bar{Y}$ in $\overline{\mathcal{M}}_{3, V}$ for each of the possibilities for the decorated graph $(\bar{X}, \bar{D})$. Recall from the beginning of Section 2.3 that the image in $\overline{\mathcal{M}}_{3, V}$ of the semistable curve $\bar{Y}$ is obtained by contracting all its irreducible components of genus 0 which intersect the rest of $\bar{Y}$ in at most two points. We discuss one of the cases. The other cases are similar. The different types are depicted in Figure A.2 in Appendix A. 
Let $(\bar{X}, \bar{D})$ be a decorated graph of type III.5, Figure 2.1 .

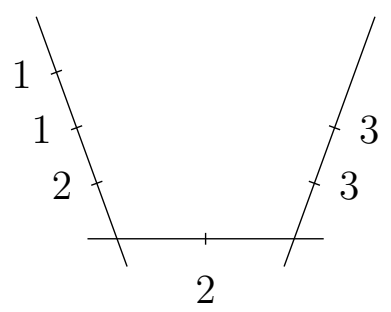

Figure 2.1. $\bar{X}$ of Type III.5.

We number the irreducible components of $\bar{X}$ as $\bar{X}_{1}, \bar{X}_{2}, \bar{X}_{3}$ (from left to right). Let $\bar{f}: \bar{Y} \rightarrow \bar{X}$ be the (unique) admissible $V$-Galois cover from Lemma 2.10. By Lemma 2.9 the label of the intersection point of $\bar{X}_{1}$ and $\bar{X}_{2}$ is 2 ; the label of the intersection point of $\bar{X}_{2}$ and $\bar{X}_{3}$ is 0 .

The restriction of $\bar{f}$ to $\bar{X}_{1}$ contains branch points with two different inertia generators. Therefore there is a unique irreducible component $\bar{Y}_{1}$ of $\bar{Y}$ above $\bar{X}_{1}$. The RiemannHurwitz formula implies that $\bar{Y}_{1}$ has genus 1 . All branch points of the restriction of $\bar{f}$ to $\bar{X}_{2}$ (respectively $\bar{X}_{3}$ ) have the same label. Therefore there are two irreducible components of $\bar{Y}$ above $\bar{X}_{2}$ and two above $\bar{X}_{3}$. The Riemann-Hurwitz formula implies that all four components have genus zero. Since $\bar{f}$ is unbranched at the intersection point of $\bar{X}_{2}$ and $\bar{X}_{3}$, these four components intersect in four points as depicted in Figure 2.2 .

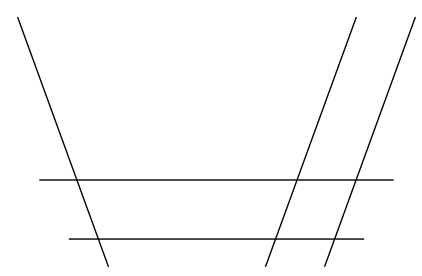

Figure 2.2. $\bar{Y}$ corresponding to Type III.5.

We conclude that there are exactly two irreducible components of $\bar{Y}$ of genus zero that intersect the rest of $\bar{Y}$ in at most two points, namely the two components above $\bar{X}_{3}$. Contracting these yields the image of $\bar{Y}$ in $\overline{\mathcal{M}}_{3, V}$ as depicted in Figure 2.3.

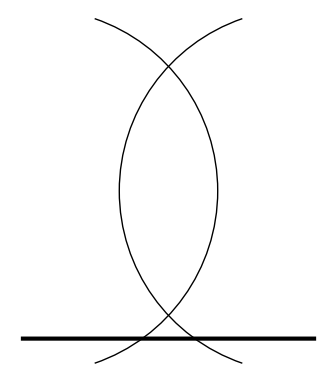

Figure 2.3. Special fiber of the stable model.

Remark 2.12. We note that none of the singular reduction types occurring in Theorem 2.11 is of compact type. Phrased differently, all the singular curves in Appendix A have loops. This is not surprising, as the inertia group of a singular point of $\bar{Y}$ is cyclic since the residue characteristic is different from 2 . One may deduce from the fact that $\bar{Y}$ is connected, that we always have loops. 
2.4. Computing the stable reduction. In this section we outline the method we use to compute the decorated graph associated with a curve $Y / K$ endowed with an action of $V$ such that all degree-2 subcovers have genus 1. We assume that $K$ is sufficiently large such that all branch points of $f: Y \rightarrow X:=Y / V$ are $K$-rational. Recall that we may assume that $Y$ is given by Equation (2.1). As in the proof of Theorem 2.11, it suffices to determine the decorated graph associated with the special fiber $(\bar{X}, \bar{D})$ of the stably marked model $(\mathcal{X}, \mathcal{D})$ of $(X, D)$, where $D$ is the branch locus of the natural map $f: Y \rightarrow X$ marked by the inertia generators.

To compute $(\bar{X}, \bar{D})$ we follow the strategy from [BW17, Section 4.2]. Loc. cit. treats the superelliptic case, i.e., the case of cyclic covers of the projective line. With our preparations, the adaptation to the current set-up is straightforward.

The key idea used in [BW17, Section 4.2] is to describe the irreducible components of $\bar{X}$ in terms of coordinates $\xi: X \stackrel{\sim}{\rightarrow} \mathbb{P}_{K}^{1}$. Every coordinate $\xi$ defines a model $\mathbb{P}_{\mathcal{O}}^{1}$ of $X$, and hence by reduction, a projective line $\bar{X}_{\xi}$ over $k$ ([BW17, Prop. 4.2.(1)]). Let $T$ be the set of triples of pairwise distinct points of $D$. Every $t=(P, Q, R) \in T$ defines a unique coordinate $\xi_{t}$ with

$$
\xi_{t}(P)=0, \xi_{t}(Q)=1, \xi_{t}(R)=\infty .
$$

Two coordinates $\xi_{1}$ and $\xi_{2}$ are equivalent if $\xi_{1} \circ \xi_{2}^{-1}: \mathbb{P}_{K}^{1} \stackrel{\sim}{\rightarrow} \mathbb{P}_{K}^{1}$ extends to an isomorphism over $\mathcal{O}$. This equivalence relation defines an equivalence relation on $T$ denoted by $\sim$. [BW17, Prop. 4.2.(3)] states that there is a bijection between $T / \sim$ and the set of irreducible components of $\bar{X}$. We write $\xi_{t}$ (respectively $\bar{X}_{t}$ ) for the coordinate (respectively the irreducible component of $\bar{X}$ ) corresponding to $t \in T$. In [BW17, Remark 4.3] it is explained how to reconstruct the intersection points between the different irreducible components from the values $\xi_{t}(P)$, where $t \in T / \sim$ and $P \in D$ runs over the branch points.

Example 2.13. Let $Y / K$ be a plane quartic curve defined by (2.1), and assume that $\nu(\Delta(X))=0, \nu(A), \nu(B)>0, \nu(C)=0, \nu(a)>0$, and $\nu(b)=\nu(c)=0$. We determine the possible stable reductions for this case. This is a special case of case (f.vi) of Theorem 3.7; the result there is formulated more symmetrically in terms of the invariants we introduce in Section 3.1.

We let $\mathcal{X}_{0}$ be the model of a conic $X$ defined by Equation (2.2) and use the notation of 2.5 for the branch points of $f: Y \rightarrow X$. Write $\beta$ (respectively $\gamma$ ) for the root of $p_{b}$ (respectively $p_{c}$ ) of valuation zero, with $p_{b}, p_{c}$ defined as in (2.3). With this convention the branch points $P_{a}, P_{a}^{\prime}$ and $P_{c}^{\prime}$ reduce to the point $(0: 1: 0)$, the branch points $P_{b}^{\prime}$ and $P_{c}$ reduce to the point $(1: 0: 0)$, and $P_{b}$ reduces to $(1: 0:-b)$ on the special fiber $\bar{X}_{0}$ of $\mathcal{X}_{0}$. In particular, $\mathcal{X}_{0}$ is not stably marked. The previous discussion implies that $\bar{X}_{0}$ is one of the irreducible components of the special fiber $\bar{X}$ of the stably marked model.

Inspection of the cases in Appendix A implies that the decorated graph $(\bar{X}, \bar{D})$ is of type IV.2, IV.3 or III.6. In order to determine the reduction type of $\bar{X}$, it suffices to distinguish between these three cases. We can do this by considering the coordinate $\xi=\xi_{t}$ of $X$ corresponding to $t=\left(P_{a}^{\prime}, P_{a}, P_{b}^{\prime}\right)$. This coordinate is given by

$$
\xi=\frac{\beta u+\alpha v+2 C w}{2(\alpha-a) \cdot v} .
$$

Here $u, v, w$ are the coordinates of $X$ as in 2.2.

Using the assumptions on the valuations of the parameters $A, B, C, a, b, c$ one computes that $\overline{\xi\left(P_{b}^{\prime}\right)}=\overline{\xi\left(P_{c}\right)}=\infty$. Hence the type of $\bar{X}$ only depends on $\overline{\xi\left(P_{c}^{\prime}\right)}$. One may check 
that $\overline{\xi\left(P_{b}\right)}=\infty$ as well, by finding a different expression for $\xi$. However, this is not needed to distinguish between the possibilities for $\bar{X}$.

Namely, $P_{a}, P_{a}^{\prime}$, and $P_{c}^{\prime}$ specialize to pairwise distinct points of the component $\bar{X}_{\xi}$ different from the intersection point of $\bar{X}_{\xi}$ with the rest of $\bar{X}$ if and only if $\overline{\xi\left(P_{c}^{\prime}\right)} \notin$ $\{0,1, \infty\}$. Otherwise, we need an additional coordinate $\xi^{\prime}$ to separate $P_{c}^{\prime}$ from the point $Q \in\left\{P_{a}, P_{a}^{\prime}, P_{b}\right\}$ with $\xi\left(P_{c}^{\prime}\right) \equiv \xi(Q)(\bmod \pi)$. However, to decide what decorated graph occurs it suffices to know for which point $Q$ this holds. It is not necessary to calculate the coordinate $\xi^{\prime}$ explicitly. The possible configurations of the components $\bar{X}_{0}$ and $\bar{X}_{\xi}$ are depicted in Figure 2.4 .
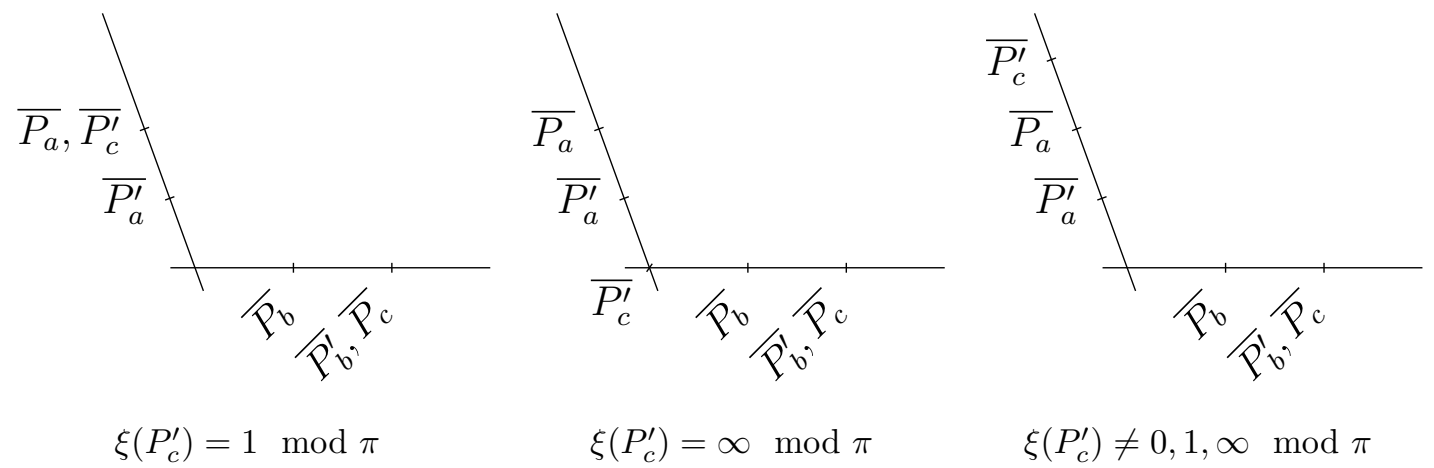

Figure 2.4. Possible configurations of $\bar{X}_{\xi}$ and $\bar{X}_{0}$.

It is no restriction to assume that $\alpha$ is the root of $p_{a}$ with smallest valuation. It follows that $\nu(\alpha) \leq \nu(a)$ and $2 \nu(\alpha) \leq \nu(B C)$. Using that $\beta \equiv 2 b(\bmod \pi)$ and $\gamma \equiv 2 c(\bmod \pi)$ are units in $\mathcal{O}$, we find

$$
\xi\left(P_{c}^{\prime}\right)=\frac{-2 B \beta+\alpha \gamma}{2(\alpha-a) \gamma} \equiv \frac{\alpha(2 a b-b \alpha-2 c C)}{4 c C(a-\alpha)} \quad(\bmod \pi) .
$$

By our assumptions, we have that $\nu(\alpha-a) \geq \nu(\alpha)$ and $2 a b-b \alpha-2 c$ is a unit. Hence we get $\overline{\xi\left(P_{c}^{\prime}\right)} \neq 0$. There remain three cases:

$$
\overline{\xi\left(P_{c}^{\prime}\right)}= \begin{cases}\overline{\xi\left(P_{a}\right)}=1 & \text { iff } \nu(B C)>2 \nu(a), \text { (type IV.3), } \\ \overline{\xi\left(P_{b}\right)}=\infty & \text { iff } \nu\left(\Delta_{a}\right)>\nu(B C)=2 \nu(a),(\text { type IV.2) } \\ \neq 0,1, \infty & \text { otherwise, (type III.6). }\end{cases}
$$

One may check that the last case occurs if and only if $\nu\left(\Delta_{a}\right)=\nu(B C)<2 \nu(a)$.

As in the proof of Theorem 2.11 one finds that the reduction type of $\bar{Y}$ is Winky Cat if $\bar{X}$ is of type III.6, Cat if $\bar{X}$ is of type IV.3, and Garden if $\bar{X}$ is of type IV.2.

If $\bar{X}$ is of type III.6, then the curve $\bar{Y}$ has one component $\bar{Y}_{1}$ of positive genus. The curve $\bar{Y}_{1}$ is an elliptic curve and the restriction of $\bar{f}$ to $\bar{Y}_{1}$ factors as $\bar{Y}_{1} \rightarrow \bar{X}_{\xi^{\prime}} \rightarrow \bar{X}_{\xi}$, where $\bar{X}_{\xi^{\prime}}:=\bar{Y}_{1} /\left\langle\sigma_{c}\right\rangle$ is a projective line. Lemma 2.9 yields a description of the inertia generators. We explain how to compute the $j$-invariant of $\bar{Y}_{1}$.

The map $\bar{X}_{\xi^{\prime}} \rightarrow \bar{X}_{\xi}$ has degree 2 and is exactly branched at $\overline{\xi\left(P_{a}^{\prime}\right)}=0$ and $\overline{\xi\left(P_{a}\right)}=1$. Normalizing the unique point of $\bar{X}_{\xi^{\prime}}$ above $P_{a}^{\prime}$ (respectively $P_{a}$ ) to 0 (respectively 1 ) and one of the points above the intersection point of $\bar{X}_{\xi}$ with the rest of $\bar{X}$ to $\infty$, we obtain

$$
\bar{X}_{\xi^{\prime}} \rightarrow \bar{X}_{\xi}, \quad \xi^{\prime} \mapsto \xi=\frac{\left(\xi^{\prime}\right)^{2}}{2 \xi^{\prime}-1} .
$$


The degree-2 map $\bar{Y}_{1} \rightarrow \bar{X}_{\xi^{\prime}}$ is branched at the inverse image of $\xi\left(P_{c}^{\prime}\right)=: \lambda$ and $\xi=\infty$, i.e., at $\xi^{\prime}=\infty, 1 / 2$ and the roots of $t^{2}-2 \lambda t+\lambda$. Using the assumptions on the parameters we find that $\lambda=\alpha / 2(\alpha-a)$. Taking the cross ratio of these 4 points, we find that

$$
j\left(\bar{Y}_{1}\right) \equiv \frac{2^{6}\left(a^{2}+12 B C\right)^{3}}{\Delta_{a}^{2} \cdot 4 B C} \quad(\bmod \pi) .
$$

Remark 2.14. $\quad$ (a) In Proposition 3.1 we formulate certain minimality conditions of the parameters $A, B, C, a, b, c$. Assuming these conditions, Equation (2.2) defines a model of $X$, which we denote by $\mathcal{X}_{0}$. In the situation of Example 2.13 this model is semistable. However, this is not true in general. It may happen that the special fiber $\bar{X}_{0}$ of $\mathcal{X}_{0}$ is not reduced. In Proposition 4.9.(ii) this case is characterized. The method to compute the stable model of $X$ still works, but one need to pass to a further extension of $K$ to find a model of $X$ whose special fiber is reduced. More details can be found in the proof of Lemma 4.12 .

(b) In Example 2.13 we sketched a systematic method for computing the invariants of the components of positive genus of the stable reduction of a curve $Y$. This method has the advantage that it always works. Once one knows the type of the stable reduction of a curve $Y$, it is sometimes faster to explicitly write down a model $\mathcal{Y}$ of $Y$, that is not necessarily semistable, to calculate the invariants of the components of positive genus of the stable reduction of a curve $Y$.

Namely, let $\mathcal{Y}$ be a model, that is not necessarily semistable, of $Y$ and assume that the normalization of its reduced special fiber contains an irreducible component $Z$ of positive genus. Then the uniqueness of the stable model implies that $Z$ is also an irreducible component of the normalization of the stable reduction $\bar{Y}^{\text {stab }}$ of $Y$. Therefore an equation for $Z$ may be used to compute the invariants for the corresponding irreducible component of the stable reduction. This method is used, for example, in the proof of Lemma 4.2 . We refer to this proof for more details.

\section{Smooth Plane QUaRTic CASe}

Let $Y / K$ be a smooth projective plane quartic over a complete discrete valuation field of characteristic 0 and residue charactertistic $2 \neq p \geq 0$, such that $\operatorname{Aut}_{\bar{K}}(Y)$ contains a subgroup $V \simeq C_{2} \times C_{2}$. Recall that, by Lemma 2.2, $Y$ admits an equation of the form (2.1):

$$
Y: A x^{4}+B y^{4}+C z^{4}+a y^{2} z^{2}+b z^{2} x^{2}+c x^{2} y^{2}=0
$$

for some $A, B, C, a, b, c \in K$, possibly after replacing $K$ by a finite extension.

In Section 3.2 we identify the reduction type of a given curve $Y / K$, where all possible types are listed in Appendix A. Before stating the results we discuss the problem that an equation of the form (2.1), and hence the coefficients $A, B, C, a, b, c$, for $Y / K$ are only unique up to $K$-isomorphisms. Proposition 3.1 states a normalization condition for the valuation of the coefficients of (2.1). This allows us in Proposition 3.2 to exhibit a set of invariants $I_{3}, I_{3}^{\prime}, I_{3}^{\prime \prime}, I_{6}$ for the special locus $\mathcal{M}_{3, V}^{\text {quar }}$. Proposition 3.1 allows us to assume that $I_{3}, I_{3}^{\prime}, I_{3}^{\prime \prime}, I_{6}$ have non-negative valuation and at least one has valuation zero. The classification of the reduction types of $Y$ in terms of the invariants is stated in Theorems 3.7 and 3.8 .

3.1. Invariants. Let $Y / K$ be given as in the form (2.1); such equation can be normalized, as given by the following result: 
Proposition 3.1. After a suitable change of variables in (2.1) we can always assume that the valuation of at least one of the elements in each set $\{A, B, c\},\{A, b, C\},\{a, B, C\}$, $\{A, b, c\},\{a, B, c\},\{a, b, C\}$ is zero while all the others are non-negative.

Proof. Let $\pi(r), \pi(s), \pi(t) \in \mathcal{O}$ be elements with valuation $r, s$, and $t$ respectively. Observe that the change of variables

$$
(x, y, z) \mapsto(\pi(r) x, \pi(s) y, \pi(t) z)
$$

changes the valuation of the coefficients as follows

$$
\begin{aligned}
& \nu(A) \mapsto \nu(A)+4 r, \quad \nu(B) \mapsto \nu(B)+4 s, \quad \nu(C) \mapsto \nu(C)+4 t, \\
& \nu(a) \mapsto \nu(a)+2 s+2 t, \quad \nu(b) \mapsto \nu(b)+2 r+2 t, \quad \nu(c) \mapsto \nu(c)+2 r+2 s .
\end{aligned}
$$

Let $U$ be one of the sets listed in the statement, and assume that all the valuations of the parameters in $U$ are positive. We will see how to obtain an isomorphic model for $Y$ that is normalized with respect to all the sets for which the original model was normalized, and also with respect to $U$.

By symmetry it is enough to consider the cases where $U$ is $\{A, b, c\}$ or $\{A, B, c\}$.

- For the first case consider the change

$$
(x, y, z) \mapsto\left(x / \pi_{1}, y, z\right)
$$

with $\pi_{1} \in \mathcal{O}$ an element of valuation $\nu\left(\pi_{1}\right)=\min (\nu(A) / 4, \nu(b) / 2, \nu(c) / 2)$. With this change, the valuation of at least one among $A, b, c$ becomes zero, and the valuations of $a, B$, and $C$ remain the same.

- Assume now that the model is normalized with respect to every set of the form $\{L, m, n\}$, and assume that it is not with respect to $\{A, B, c\}$. It follows that $\nu(a)=\nu(b)=0$, since otherwise we could normalize further with respect to either $\{a, B, c\}$ or $\{A, b, c\}$. Consider now the change

$$
(x, y, z) \mapsto\left(x / \pi_{2}, y / \pi_{2}, \pi_{2} z\right)
$$

with $\pi_{2} \in \mathcal{O}$ an element of valuation $\nu\left(\pi_{2}\right)=\min (\nu(A) / 4, \nu(B) / 4, \nu(c) / 2)$. With this change, the valuation of at least one among $A, B, c$ becomes zero, the valuations of $a$ and $b$ remain the same, and the valuation of $C$ increases by $4 \nu\left(\pi_{2}\right)$. The increase of the valuation of $C$ does not affect the normalization with respect to any other set, since any set containing $C$ also contains $a$ or $b$, which have valuation zero, as explained above.

Dixmier-Ohno invariants, see [Dix87, Ohn07], classify isomorphism classes of plane quartics. Moreover, in [LRS18] a reconstruction method is presented to compute the equation of a curve corresponding to a given tuple of Dixmier-Ohno invariants. In [LRS16, Function IsInstrataD4] the expressions for Dixmier-Ohno invariants for quartics in the locus $\mathcal{M}_{3}^{\text {quar }}$ are given. However, it is more convenient to work with a smaller set of invariants, specifically for the locus $\mathcal{M}_{3, V}^{\text {quar }}$ instead of the general Dixmier-Ohno invariants; indeed explicit computation with these is unnecessarily complicated. We therefore consider the four invariants as in the following statement.

Proposition 3.2. The elements

$$
\begin{array}{r}
I_{3}=A B C, \quad I_{3}^{\prime}=A \Delta_{a}+B \Delta_{b}+C \Delta_{c}, \\
I_{3}^{\prime \prime}=-4 A B C+A a^{2}+B b^{2}+C c^{2}-a b c, \quad I_{6}=\Delta_{a} \Delta_{b} \Delta_{c}
\end{array}
$$

are invariants for the locus $\mathcal{M}_{3, V}^{\text {quar }}$. 
Proof. By Lemma 2.2.(1), any isomorphism between plane quartics in $\mathcal{M}_{3, V}$ has to preserve the automorphism group

$$
V \simeq\left\langle\left(\begin{array}{ccc}
-1 & 0 & 0 \\
0 & -1 & 0 \\
0 & 0 & 1
\end{array}\right),\left(\begin{array}{ccc}
-1 & 0 & 0 \\
0 & 1 & 0 \\
0 & 0 & -1
\end{array}\right)\right\rangle
$$

Since plane quartics are given by their canonical models, isomorphisms between them are linear. Commuting with the previous group implies that isomorphisms of plane quartics in $\mathcal{M}_{3, V}^{\text {quar }}$ are given by products of permutation matrices and diagonal matrices. Hence the elements from the statement of the lemma considered as element of $K[A, B, C, a, b, c]$ are invariants for the locus $\mathcal{M}_{3, V}^{\text {quar }}$.

Remark 3.3. Notice that $I_{3}^{\prime \prime}=\Delta(X)$.

Proposition 3.4. The invariants $I_{3}, I_{3}^{\prime}, I_{3}^{\prime \prime}$ and $I_{6}$ are generators for the invariants algebra of the locus $\mathcal{M}_{3, V}^{\text {quar }}$.

Proof. While in characteristic 0 a direct computation of the Dixmier-Ohno invariants in terms of these invariants (see [BCK ${ }^{+} 20$, InvariantsGenerateD0] for the details) gives the result, in positive odd characteristic we have to use another strategy.

In general, for any characteristic different from 2, we can proceed as follows: first, we normalize to obtain $A=B=C=1$ so the group $G$ acting in $K[a, b, c]$ is finite. Indeed,

$$
G=\left\langle\left(\begin{array}{lll}
0 & 0 & 1 \\
1 & 0 & 0 \\
0 & 1 & 0
\end{array}\right),\left(\begin{array}{lll}
0 & 1 & 0 \\
1 & 0 & 0 \\
0 & 0 & 1
\end{array}\right),\left(\begin{array}{ccc}
i & 0 & 0 \\
0 & i & 0 \\
0 & 0 & -1
\end{array}\right)\right\rangle \text {. }
$$

Then, we compute the invariants $K[a, b, c]^{G}$ with Magma [BCP97], and we obtain generators: $1, a^{2}+b^{2}+c^{2}, a b c, a^{2} b^{2}+a^{2} c^{2}+b^{2} c^{2}$. After de-normalizing we get the weight 3, 3, 3, 6 invariants $A B C, A a^{2}+B b^{2}+C c^{2}, a b c, A B a^{2} b^{2}+B C b^{2} c^{2}+C A c^{2} a^{2}$. Notice that in order to do the computations in Magma we needed to fix the field, we fixed the fields $\mathbb{F}_{3}$ and $\mathbb{Q}$ because the order of $G$ is a product of a power of 2 and a power of 3. Finally, the result extends to any characteristic different from 2 because of Molien's Formula ([DK02, Theorem 3.2.2]).

The invariants $I_{3}, I_{3}^{\prime}, I_{3}^{\prime \prime}, I_{6}$ are homogeneous of weight $3,3,3,6$, respectively. Moreover, considered as functions on the weighted projective space $\mathbb{P}_{3,3,3,6}^{3}$, they are algebraically independent. To state the classification theorems for the reduction types of plane quartics it is also convenient to define

$$
I=A B \Delta_{a} \Delta_{b}+A C \Delta_{a} \Delta_{c}+B C \Delta_{b} \Delta_{c} .
$$

The invariant $I$ is in the algebra generated by $I_{3}, I_{3}^{\prime}, I_{3}^{\prime \prime}, I_{6}$. Concretely, we have

$$
4 I+I_{6}-I_{3}^{\prime 2}+16 I_{3} I_{3}^{\prime \prime}+2 I_{3}^{\prime} I_{3}^{\prime \prime}-I_{3}^{\prime \prime 2}=0 .
$$

We now restate Proposition 3.1 in terms of these invariants:

Corollary 3.5. After a change of variables as in Proposition 3.1 we can always work with an integer model as in (2.1) such that all the valuations of $I_{3}, I_{3}^{\prime}, I_{3}^{\prime \prime}$ and $I_{6}$ are non-zero and at least one is equal to zero.

Proof. Suppose that all four invariants have positive valuations. Then we have $\nu\left(I_{3}\right)=$ $\nu(A B C)>0$, and without loss of generality we can assume that $\nu(A)$ is positive. Then, since $\nu\left(I_{3}^{\prime}\right), \nu\left(I_{3}^{\prime \prime}\right)$ and $\nu\left(I_{6}\right)$ are positive, we obtain $\nu\left(B b^{2}+C c^{2}\right), \nu(a b c), \nu\left(B C b^{2} c^{2}\right)>0$, respectively. So we are in one of the following scenarios: 
- $\nu(B), \nu(C), \nu(a b c)>0$, or

- $\nu(B), \nu(c)>0$ or, symmetrically, $\nu(C), \nu(b)>0$, or

- $\nu(b), \nu(c)>0$,

but all contradict the normalization conditions in Proposition 3.1. Hence the corollary follows.

3.2. Main results. In this section we characterize the possible reduction types of a plane quartic curve $Y$ in terms of the valuations of the four invariants $I_{3}, I_{3}^{\prime}, I_{3}^{\prime \prime}, I_{6}$. We assume that $Y$ is given by an equation of the form $A x^{4}+B y^{4}+C z^{4}+a y^{2} z^{2}+b x^{2} z^{2}+c x^{2} y^{2}=0$, normalized as in Proposition 3.1; in particular by Corollary 3.5 all the invariants have non-negative valuation, and at least one of them has valuation zero. Additionally, we use the invariant $I$ determined by (3.1).

In terms of the invariants, we have

$$
\Delta(Y)=-2^{-20} I_{3} I_{3}^{\prime \prime 4} I_{6}^{2} .
$$

Proposition 3.6. Let $Y$ be a plane quartic defined by

$$
A x^{4}+B y^{4}+C z^{4}+a y^{2} z^{2}+b x^{2} z^{2}+c x^{2} y^{2}=0
$$

normalized as in Proposition 3.1. Let $\Delta(Y)$ be the discriminant of $Y$. The plane quartic $Y$ has potentially good reduction if and only if $\nu(\Delta(Y))=0$. Equivalently, if and only if $\nu\left(I_{3}\right)=\nu\left(I_{3}^{\prime \prime}\right)=\nu\left(I_{6}\right)=0$.

Proof. If $\nu(\Delta(Y))=0$, then the curve has good reduction, hence it has potentially good reduction. If $Y$ has potentially good reduction then over a finite extension of the base field, it admits a plane quartic integral model with good reduction, and because of Corollary 3.5 in [LLLR19, this model can be taken in the form

$$
A x^{4}+B y^{4}+C z^{4}+a y^{2} z^{2}+b x^{2} z^{2}+c x^{2} y^{2}=0
$$

with $A, B, C, a, b, c \in \mathcal{O}, \nu(\Delta(Y))=0$. In particular, with $\nu\left(I_{3}\right), \nu\left(I_{3}^{\prime}\right), \nu\left(I_{3}^{\prime \prime}\right), \nu\left(I_{6}\right) \geq 0$ and hence with $\nu\left(I_{3}\right)=\nu\left(I_{3}^{\prime \prime}\right)=\nu\left(I_{6}\right)=0$.

In what follows we assume that $Y$ has geometric bad reduction. The result is divided into two statements, depending on whether $\nu\left(I_{3}^{\prime \prime}\right)$ is zero (Theorem 3.7) or positive (Theorem 3.8). Recall from Remark 3.3 that $I_{3}^{\prime \prime}$ is the discriminant of the conic $X$ defined by (2.2). Hence the two cases correspond to the reduction of this conic being non-degenerate or degenerate (see also Remark 2.14.(b)).

Theorem 3.7 is proved in Section 4.1 and Theorem 3.8 in Section 4.2. In these section we also give the Igusa invariants (respectively the $j$-invariant) of the irreducible components of the stable reduction of $Y$ with positive genus in each of the cases.

Theorem 3.7. Let $Y$ be a plane quartic curve defined by

$$
A x^{4}+B y^{4}+C z^{4}+a y^{2} z^{2}+b x^{2} z^{2}+c x^{2} y^{2}=0
$$

normalized as in Proposition 3.1. Let $\Delta(Y)$ be the discriminant of the quartic $Y$, and let $\Delta(X)$ be the discriminant of the conic $X$, which we assume to have valuation 0 , that is, $\nu\left(I_{3}^{\prime \prime}\right)=0$.

Then if the valuation of $\Delta(Y)$ is positive, $Y$ has geometric bad reduction and one of the cases in Table 3.1 occurs.

Theorem 3.8. Let $Y$ be a plane quartic curve defined by

$$
A x^{4}+B y^{4}+C z^{4}+a y^{2} z^{2}+b x^{2} z^{2}+c x^{2} y^{2}=0
$$




\begin{tabular}{|c|c|c|c|c|c|c|c|c|c|}
\hline & $\nu\left(I_{3}\right)$ & $\nu\left(I_{3}^{\prime}\right)$ & $\nu\left(I_{3}^{\prime \prime}\right)$ & $\nu\left(I_{6}\right)$ & $\nu(I)$ & Other conditions & Decorated graphs & Stable curve & Lemma \\
\hline (a) & $=0$ & & $=0$ & $>0$ & $=0$ & & II.3 & Loop & \\
\hline (b) & $=0$ & $=0$ & $=0$ & $>0$ & $>0$ & & III.1 & DNA & 4.2 \\
\hline (c) & $=0$ & $>0$ & $=0$ & $>0$ & $>0$ & & $\mathrm{IV}^{*} .1$ & Braid & \\
\hline (d) & $>0$ & $=0$ & $=0$ & $=0$ & $=0$ & & II.4 & Lop & 4.4 \\
\hline (e) & $>0$ & $=0$ & $=0$ & $>0$ & $=0$ & & III.2 & Looop & 4.5 \\
\hline (f.i) & & & & & & $\begin{array}{c}2 \nu(I)>\nu\left(I_{3}\right)+\nu\left(I_{6}\right)>2 \nu\left(I_{3}\right) \\
\quad \text { or } \nu\left(I_{3}\right)<\nu(I)<\nu\left(I_{6}\right)\end{array}$ & IV.1 & Grl Pwr & \\
\hline (f.ii) & & & & & & $\begin{array}{c}2 \nu(I)>\nu\left(I_{3}\right)+\nu\left(I_{6}\right)>2 \nu\left(I_{6}\right) \\
\quad \text { or } \nu\left(I_{3}\right)>\nu(I)>\nu\left(I_{6}\right)\end{array}$ & IV.3 & Cat & \\
\hline (f.iii) & $>0$ & $=0$ & $=0$ & $>0$ & $>0$ & $\begin{array}{c}2 \nu(I)>\nu\left(I_{3}\right)+\nu\left(I_{6}\right)=2 \nu\left(I_{3}\right) \\
\quad \text { or } \nu\left(I_{3}\right)=\nu(I)=\nu\left(I_{6}\right)\end{array}$ & II.1 & Candy & 4.6 \\
\hline (f.iv) & & & & & & $\nu(I)<\nu\left(I_{3}\right), \nu(I)<\nu\left(I_{6}\right)$ & IV.2 & Garden & \\
\hline (f.v) & & & & & & $\nu(I)=\nu\left(I_{3}\right)<\nu\left(I_{6}\right)$ & III.5 & Tree & \\
\hline (f.vi) & & & & & & $\nu(I)=\nu\left(I_{6}\right)<\nu\left(I_{3}\right)$ & III.6 & Winky Cat & \\
\hline$(g)$ & $>0$ & $=0$ & $=0$ & $=0$ & $>0$ & & III.3 & Loop & 4.7 \\
\hline (h) & $>0$ & $>0$ & $=0$ & $=0$ & $>0$ & & $\mathrm{IV}^{*} .3$ & Looop & 4.8 \\
\hline
\end{tabular}

TABle 3.1. Cases of Theorem 3.7.

normalized as in Proposition 3.1. Let $\Delta(Y)$ be the discriminant of the quartic $Y$, and let $\Delta(X)$ be the discriminant of the conic $X$, which we assume to have positive valuation, that is, $\nu\left(I_{3}^{\prime \prime}\right)>0$.

Then the valuation of $\Delta(Y)$ is positive, $Y$ has geometric bad reduction and one of the cases in Table 3.2 occurs.

\begin{tabular}{|c|c|c|c|c|c|c|c|c|c|}
\hline & $\nu\left(I_{3}\right)$ & $\nu\left(I_{3}^{\prime}\right)$ & $\nu\left(I_{3}^{\prime \prime}\right)$ & $\nu\left(I_{6}\right)$ & $\nu(I)$ & Other conditions & Decorated graphs & Stable curve & Lemma \\
\hline (a) & $=0$ & & $>0$ & $=0$ & & & II.2 & DNA & 4.10 \\
\hline (b.i) & \multirow{3}{*}{$\nu\left(I_{3}+I\right.$} & \multirow{3}{*}{$\left.I_{3}^{\prime}\right)=0$} & \multirow{3}{*}{$>0$} & \multirow{3}{*}{$>0$} & \multirow{3}{*}{$=0$} & $0<\nu\left(I_{3}^{\prime \prime}\right)<\nu\left(I_{6}\right)$ & $\mathrm{IV}^{*} .2$ & DNA & \multirow{3}{*}{4.11} \\
\hline (b.ii) & & & & & & $\nu\left(I_{3}^{\prime \prime}\right)>\nu\left(I_{6}\right)>0$ & IV.5 & Braid & \\
\hline (b.iii) & & & & & & $\nu\left(I_{3}^{\prime \prime}\right)=\nu\left(I_{6}\right)>0$ & III.4 & Candy & \\
\hline (c.i) & \multirow{7}{*}{$=0$} & \multirow{7}{*}{$>0$} & \multirow{7}{*}{$>0$} & \multirow{7}{*}{$>0$} & \multirow{7}{*}{$>0$} & $\begin{array}{c}\nu\left(I_{6}^{2}\right)=\nu\left(I_{3} I_{3}^{\prime \prime 3}\right) \\
\nu\left(I_{3}^{\prime 4}\right) \geq \nu\left(I_{3} I_{3}^{\prime \prime}\right)\end{array}$ & I & Good (hyp) & \\
\hline (c.ii) & & & & & & $\begin{array}{c}\nu\left(I_{6}^{2}\right)>\nu\left(I_{3} I_{3}^{\prime \prime 3}\right) \\
\nu\left(I_{3}^{\prime 4}\right) \geq \nu\left(I_{3} I_{3}^{\prime \prime}\right), \text { and } \\
\nu\left(I_{3}^{\prime 4}-4 I_{3} I_{3}^{\prime \prime}\right)=\nu\left(I_{3} I_{3}^{\prime \prime}\right)\end{array}$ & II.3 & Loop (hyp) & \\
\hline (c.iii) & & & & & & $\begin{array}{c}\nu\left(I_{6}^{2}\right)>\nu\left(I_{3} I_{3}^{\prime \prime 3}\right) \\
\nu\left(I_{3}^{\prime 4}\right)=\nu\left(I_{3} I_{3}^{\prime \prime}\right), \text { and } \\
\nu\left(I_{3}^{\prime 4}-4 I_{3} I_{3}^{\prime \prime}\right)>\nu\left(I_{3} I_{3}^{\prime \prime}\right)\end{array}$ & III.1 & DNA (hyp) & 4.12 \\
\hline (c.iv) & & & & & & $\begin{array}{c}\nu\left(I_{6}^{2}\right)<\nu\left(I_{3} I_{3}^{\prime \prime 3}\right) \text { and } \\
\nu\left(I_{3}^{\prime 4}\right) \geq \nu\left(I_{3} I_{6}\right)\end{array}$ & II. 2 & DNA (hyp) & \\
\hline (c.v) & & & & & & $12 \nu\left(I_{3}^{\prime}\right)<3 \nu\left(I_{3} I_{3}^{\prime \prime}\right)<2 \nu\left(I_{3} I_{6}\right)$ & $\mathrm{IV}^{*} .2$ & DNA (hyp) & \\
\hline (c.vi) & & & & & & $12 \nu\left(I_{3}^{\prime}\right)<2 \nu\left(I_{3} I_{6}\right)<3 \nu\left(I_{3} I_{3}^{\prime \prime}\right)$ & IV.5 & Braid (hyp) & \\
\hline (c.vii) & & & & & & $12 \nu\left(I_{3}^{\prime}\right)<2 \nu\left(I_{3} I_{6}\right)=3 \nu\left(I_{3} I_{3}^{\prime \prime}\right)$ & III.4 & Candy (hyp) & \\
\hline$(\mathrm{d})$ & $>0$ & & $>0$ & $=0$ & $=0$ & & III.7 & Cave & 4.13 \\
\hline (e) & $>0$ & $=0$ & $>0$ & & $>0$ & & IV.4 & Braid & 4.14 \\
\hline
\end{tabular}

TABle 3.2. Cases of Theorem 3.8.

In the following section we give a detailed proof of the two theorems, using the strategy explained in Section 2.4. In particular, for each case, we determine the special fiber $(\bar{X}, \bar{D})$ 
of the stably marked model of $(X, D)$, thus we obtain one of the twenty decorated graphs depicted in Appendix A and, as in the proof of Theorem 2.11, we deduce the corresponding stable curve, which is the special fiber of the stable model of $Y$ (Section 2.2).

Remark 3.9. The stable curve of type Candy corresponds to the decorated graphs II.1 (see Theorem 3.7.(f.iii)) and III.4 (see Theorem 3.8.(b.iii) and Theorem 3.8.(c.vii)). Here, this can really be considered as two different reduction types, since the $j$-invariants of the elliptic curves $\bar{Y}_{1}$ and $\bar{Y}_{2}$ of the stable curve behave differently.

In the first case, it is shown in Lemma 4.6 that $j\left(\bar{Y}_{1}\right)$ and $j\left(\bar{Y}_{2}\right)$ depend on the value of the invariants and are in general not the same in $k$.

In the second case, $\bar{Y}_{1}$ and $\bar{Y}_{2}$ are isomorphic and we have that $j\left(\bar{Y}_{1}\right)=j\left(\bar{Y}_{2}\right)=1728$. This is proved in Lemma 4.11 and Corollary 5.7.

The difference between the two cases may be explained by considering the action of $V$ on the stable reduction $\bar{Y}$ of $Y$. We refer to the proofs of the results for more details.

Example 3.10. As an example we treat the curve

$$
Y: 2 x^{4}+2 y^{4}+15 z^{4}-11 y^{2} z^{2}-11 x^{2} z^{2}+3 x^{2} y^{2}=0 .
$$

Its automorphism group $\operatorname{Aut}_{\mathbb{C}}(Y)$ is isomorphic to $D_{4}$. Additionally to the action of $V$, there is an automorphism $(x: y: z) \mapsto(y:-x, z)$ of order 4 . All its automorphisms are defined over $\mathbb{Q}$. We have that

$$
\Delta(X)=I_{3}^{\prime \prime}=2^{4}, \quad \Delta(Y)=2^{2} \cdot 3 \cdot 5 \cdot 7^{2} .
$$

This curve has been studied by Howe-Leprévost-Poonen ([HLP00, Cor. 16]). They show that the conductor of this curve is $N=2940=\Delta(Y)$, which is the smallest value for a curve of genus 3 that we know of. The curve is also found by Sutherland ([Sut18]) in his database of non-hyperelliptic genus-3 curves over $\mathbb{Q}$ with small discriminant. In fact, it is the curve with smallest discriminant in this database.

When we apply the results of this section to this curve for the primes $p=3,5,7$, we find that the reduction type of $Y$ is Lop for $p=3,5$ (case (d) of Theorem 3.7) and Loop for $p=7$ (case (a) of Theorem 3.7).

\section{Proofs of main Results}

4.1. Main result with non-degenerate conic. In this section we prove Theorem 3.7 , i.e., the case that the reduction of the conic $X$ is non-degenerate. In particular in this section we assume that $I_{3}^{\prime \prime}=\Delta(X)$ has valuation zero. The equation (2.2) defines a smooth model $\mathcal{X}_{0}$ of $X$ over $\mathcal{O}$. Its special fiber, which we denote by $\bar{X}_{0}$, is an irreducible component of the special fiber $\bar{X}$ of $\mathcal{X}$, where $(\mathcal{X}, \mathcal{D})$ is the stably marked model of $(X, D)$ from Proposition 2.5.(1). Recall that $D$ denotes the branch divisor of $f: Y \rightarrow X$. Moreover, the special fiber $(\bar{X}, \bar{D})$ of the stably marked model of $(X, D)$ is a decorated graph defined in Definition 2.8. In the proof of Theorem 2.11 we have seen that there are 20 possibilities for the decorated graph, which determine the 13 possibilities for the stable reduction of $Y$. The possibilities for the decorated graph and the stable reduction of $Y$ are listed in Appendix A. The strategy of the proofs is explained in Example 2.13.

We assume that $D$ splits over $K$ and use the notation $P_{a}, P_{a}^{\prime}, P_{b}, P_{b}^{\prime}, P_{c}, P_{c}^{\prime}$ as in (2.5) for the 6 branch points of $f$. The following lemma is useful in determining the cases for the decorated graph.

Lemma 4.1. If $\nu\left(\Delta_{a}\right)$ is positive, then the points $P_{a}, P_{a}^{\prime}$ specialize to the same point in $\bar{X}_{0}$. 
If $\nu(A)$ is positive, then one point among $P_{b}, P_{b}^{\prime}$ and one point among $P_{c}, P_{c}^{\prime}$ specialize to the same point in $\bar{X}_{0}$.

Proof. If $\nu\left(\Delta_{a}\right)$ is positive, then the two roots of $p_{a}(T)=T^{2}-2 a T+4 B C$ are congruent modulo $\pi$, thus $P_{a}, P_{a}^{\prime}$ specialize to the same point in $\bar{X}_{0}$.

Similarly, if $\nu(A)$ is positive, one root of $p_{b}(T)=T^{2}-2 b T+4 A C$ and one root of $p_{c}(T)=T^{2}-2 c T+4 A B$ have positive valuation, thus one point among $P_{b}, P_{b}^{\prime}$ and one point among $P_{c}, P_{c}^{\prime}$ specialize to $(1: 0: 0)$ in $\bar{X}_{0}$.

Lemma 4.2 (Theorem 3.7, cases (a)-(c)). Let $Y$ be as in Theorem 3.7, in particular $\nu\left(I_{3}^{\prime \prime}\right)=0$. Assume that $\nu\left(I_{3}\right)=0$ and $\nu\left(I_{6}\right)>0$. Then one of the following occurs:

(a) If $\nu(I)=0$, then the decorated graph has type II.3 and the reduction type of the curve is Loop, with $j$-invariant $j=16\left(16 I_{3} I_{3}^{\prime \prime}+I\right)^{3} /\left(I_{3} I_{3}^{\prime \prime} I^{2}\right)$;

(b) if $\nu\left(I_{3}^{\prime}\right)=0$ and $\nu(I)>0$, then the decorated graph has type III.1 and the reduction type of the curve is DNA; and

(c) if $\nu\left(I_{3}^{\prime}\right)>0$ and $\nu(I)>0$, then the decorated graph has type $I V^{*} .1$ and the reduction type of the curve is Braid.

Proof. From the conditions on the invariants, it follows that the valuations $\nu(A), \nu(B)$, $\nu(C)$ are zero, and in case (a) (respectively (b), (c)) we get that exactly one (respectively two, three) of the valuations $\nu\left(\Delta_{a}\right), \nu\left(\Delta_{b}\right), \nu\left(\Delta_{c}\right)$ is positive.

Lemma 4.1 implies that $P_{i}$ and $P_{i}^{\prime}$ specialize to the same point of $\bar{X}_{0}$ if and only if the valuation of $\Delta_{i}$ is positive for $i \in\{a, b, c\}$. Moreover, since we have $\nu(A B C)=0$, Lemma 4.1 implies that no two points from different pairs specialize to the same point of $\bar{X}_{0}$. Since the 6 branch points specialize to at least 3 pairwise distinct points of $\bar{X}_{0}$ it follows that $\bar{X}_{0}$ is an irreducible component of the special fiber $\bar{X}$ of the stably marked model of $(X, D)$. From this it follows that we are in one of the cases II.3, III.1 or IV*.1. We obtain that $\bar{X}$ is of type II.3 (respectively III.1 or IV*.1) if exactly one (respectively two or three) of the valuations $\nu\left(\Delta_{a}\right), \nu\left(\Delta_{b}\right), \nu\left(\Delta_{c}\right)$ are positive. As in the proof of Theorem 2.11 it follows that the stable reduction $\bar{Y}$ of $Y$ is Loop (respectively DNA or Braid).

To compute the $j$-invariant in case (a) assume that $\Delta_{a}$ is the discriminant with positive valuation. Chosing square roots $\sqrt{B}$ and $\sqrt{C}$ with $\nu(a-2 \sqrt{B C})>0$ in an extension of $K$ we obtain an equation

$$
\mathcal{X}_{0}: A u^{2}+(b w+c v) u+G_{2}(v, w)=0,
$$

where $G_{2}(v, w) \equiv(\sqrt{B} v+\sqrt{C} w)^{2}(\bmod \pi)$. Here we use that $\nu\left(\Delta_{a}\right)$ is positive.

Write $\mathcal{Y}_{0}$ for the normalization of $\mathcal{X}_{0}$ in the function field of $Y$. Its special fiber $\bar{Y}_{0}$ is birationally given by

$$
\bar{Y}_{0}: A x^{4}+\left(b z^{2}+c y^{2}\right) x^{2}+G_{2}\left(y^{2}, z^{2}\right)^{2}=0 .
$$

There exists a change of coordinates $S \in \mathrm{GL}_{3}(K)$ such that the equation for $\mathcal{Y}_{0}$ with respect to the new variables $\left(x_{1}=x, y_{1}, z_{2}\right)$ still has integral coefficients and $G_{2}(y, z) \equiv$ $y_{1} z_{1}(\bmod \pi)$. Here we use the assumption $\nu(A B C)=0$. Hence $\bar{Y}_{0}$ may birationally be given by

$$
\bar{Y}_{0}: A x_{1}^{4}+G_{3}\left(y_{1}, z_{1}\right) x_{1}^{2}+y_{1} z_{1} \equiv 0 \quad(\bmod \pi)
$$

for some polynomial $G_{3}\left(y_{1}, z_{1}\right)=a_{0} z_{1}^{2}+a_{1} z_{1} y_{1}+a_{2} y_{1}^{2} \in \mathcal{O}\left[y_{1}, z_{1}\right]$. We set $z_{1}=1$, multiply the equation by $\left(1+a_{2} x^{2}\right)$, and define $y_{2}=\left(\left(1+a_{2} x_{1}^{2}\right) y_{1}+a_{1} x_{1}^{2} / 2\right) / x_{1}$. A short calculation shows that $\bar{Y}_{0}$ is birationally given by

$$
\bar{Y}_{0}: y_{2}^{2} \equiv-\left(1+a_{2} x^{2}\right)\left(a_{0}+A x^{2}\right)+a_{1}^{2} / 4 x^{2} \quad(\bmod \pi) .
$$


This is an elliptic curve with $j$-invariant

$$
j \equiv \frac{16\left(16 I_{3} I_{3}^{\prime \prime}+I\right)^{3}}{I_{3} I_{3}^{\prime \prime} I^{2}} \quad(\bmod \pi)
$$

This expression is also valid if $\nu\left(\Delta_{b}\right)\left(\right.$ resp. $\left.\nu\left(\Delta_{c}\right)\right)$ is positive instead of $\nu\left(\Delta_{a}\right)$.

We have already seen that the stable reduction $\bar{Y}$ of $Y$ is Loop. It follows that the normalization of $\bar{Y}$ is the normalization of $\bar{Y}_{0}$. The statement on the $j$-invariant in the lemma follows.

Remark 4.3. Notice that while checking the validity of equation 4.1 is straightforward, the computation of the right hand side from the left hand side is not.

Lemma 4.4 (Theorem 3.7, case (d)). Let $Y$ be as in Theorem 3.7, in particular $\nu\left(I_{3}^{\prime \prime}\right)=0$. Assume $\nu\left(I_{3}\right)>0, \nu\left(I_{3}^{\prime}\right)=0, \nu\left(I_{6}\right)=0$ and $\nu(I)=0$. Then the decorated graph has type II.4 and the reduction type of the curve is Lop, and the Igusa invariants of the genus-2 curve are

$$
\begin{aligned}
J_{2}= & I_{3}^{\prime} I_{3}^{\prime \prime}-I_{3}^{\prime \prime 2}+2 I_{6}+24 I, \\
J_{4}= & I_{3}^{\prime \prime 2} I_{6}+64 I_{3}^{\prime} I_{3}^{\prime \prime} I-64 I_{3}^{\prime \prime 2} I+128 I_{6} I+768 I^{2}, \\
J_{6}= & I_{3}^{\prime \prime 2} I_{6} I-32 I_{3}^{\prime} I_{3}^{\prime \prime} I^{2}+32 I_{3}^{\prime \prime 2} I^{2}-64 I_{6} I^{2}-256 I^{3}, \\
J_{8}= & I_{3}^{\prime \prime 4} I_{6}^{2}+256 I_{3}^{\prime} I_{3}^{\prime \prime 3} I_{6} I-256 I_{3}^{\prime \prime 4} I_{6} I+512 I_{3}^{\prime \prime 2} I_{6}^{2} I+4608 I_{3}^{\prime \prime 2} I_{6} I^{2} \\
& -32768 I_{3}^{\prime} I_{3}^{\prime \prime} I^{3}+32768 I_{3}^{\prime \prime 2} I^{3}-65536 I_{6} I^{3}-196608 I^{4}, \\
J_{10}= & I_{3}^{\prime \prime 4} I_{6}^{2} I .
\end{aligned}
$$

Proof. From the conditions on the invariants, it follows that exactly one valuation among $\nu(A), \nu(B), \nu(C)$ is positive and that all the valuations $\nu\left(\Delta_{a}\right), \nu\left(\Delta_{b}\right), \nu\left(\Delta_{c}\right)$ are zero. Assume $\nu(A)>0$. Then, by Lemma 4.1, a point with inertia generator $\sigma_{b}$ and a point with inertia generator $\sigma_{c}$ both specialize to $(1: 0: 0)$, hence the decorated graph has type II.4 and the reduction of the curve is Lop.

We determine an equation for the normalization of the stable reduction $\bar{Y}$ of $Y$. Since we are in case Lop this is a curve of genus 2. Arguing as in the proof of Lemma 4.2, we find that $\bar{Y}$ is birationally given by

$$
\bar{Y}: x^{2} \equiv-\frac{B y^{4}+a y^{2} z^{2}+C z^{4}}{c y^{2}+b z^{2}} .
$$

Hence $t^{2}=\left(x\left(c y^{2}+b z^{2}\right)\right)^{2}=-\left(c y^{2}+b z^{2}\right)\left(B y^{4}+a y^{2} z^{2}+C z^{4}\right)$ is the genus-2 curve we are looking for. One can check that the Igusa invariants of this curve are the ones given in the statement of the lemma.

Lemma 4.5 (Theorem 3.7, case (e)). Let $Y$ be as in Theorem 3.7, in particular $\nu\left(I_{3}^{\prime \prime}\right)=0$. Assume $\nu\left(I_{3}\right)>0, \nu\left(I_{3}^{\prime}\right)=0, \nu\left(I_{6}\right)>0$ and $\nu(I)=0$. Then the decorated graph has type III. 2 and the reduction type of the curve is Looop.

Proof. From the conditions on the invariants, it follows that exactly one valuation among $\nu(A), \nu(B), \nu(C)$ and exactly one among $\nu\left(\Delta_{a}\right), \nu\left(\Delta_{b}\right), \nu\left(\Delta_{c}\right)$ are positive while only one among $\nu\left(A \Delta_{a}\right), \nu\left(B \Delta_{b}\right), \nu\left(C \Delta_{c}\right)$ is also positive.

Assume $\nu\left(A \Delta_{a}\right)>0$. Then, by Lemma 4.1, the points $P_{a}$ and $P_{a}^{\prime}$ both specialize to $(0: 1:-1)$, and a point with inertia generator $\sigma_{b}$ and a point with inertia generator $\sigma_{c}$ both specialize to $(1: 0: 0)$, hence the decorated graph has type III.2 and the reduction of the curve is Looop. 
Lemma 4.6 (Theorem 3.7, case (f)). Let $Y$ be as in Theorem 3. 7, in particular $\nu\left(I_{3}^{\prime \prime}\right)=0$. Assume $\nu\left(I_{3}\right)>0, \nu\left(I_{3}^{\prime}\right)=0, \nu\left(I_{6}\right)>0$ and $\nu(I)>0$. Then one of the following occurs:

(i) If $2 \nu(I)>\nu\left(I_{3}\right)+\nu\left(I_{6}\right)>2 \nu\left(I_{3}\right)$ or $\nu\left(I_{3}\right)<\nu(I)<\nu\left(I_{6}\right)$, then the decorated graph has type IV.1 and the reduction type of the curve is Grl Pwr.

(ii) If $2 \nu(I)>\nu\left(I_{3}\right)+\nu\left(I_{6}\right)>2 \nu\left(I_{6}\right)$ or $\nu\left(I_{3}\right)>\nu(I)>\nu\left(I_{6}\right)$, then the decorated graph has type IV.3 and the reduction type of the curve is Cat.

(iii) If $2 \nu(I)>\nu\left(I_{3}\right)+\nu\left(I_{6}\right)=2 \nu\left(I_{3}\right)$ or $\nu\left(I_{3}\right)=\nu(I)=\nu\left(I_{6}\right)$, then the decorated graph has type II.1 and the reduction type of the curve is Candy, and the j-invariants of the two genus-1 components of the special fiber are the roots of the polynomial

$$
\begin{aligned}
I_{6}^{2} I_{3} I_{3}^{\prime} t^{2}-2^{4} & \left(I_{6}^{2} I+3 \cdot 2^{4} I_{3} I_{3}^{\prime} I_{6}^{2}+3 \cdot 2^{8} I_{3} I_{3}^{\prime} I_{6} I-2^{13} I_{3}^{2} I_{3}^{\prime 2} I_{6}+2^{12} I_{3} I_{3}^{\prime} I^{2}\right) t \\
+ & 2^{8}\left(I_{6}+2^{4} I+2^{8} I_{3} I_{3}^{\prime}\right)^{3}
\end{aligned}
$$

(iv) If $\nu(I)<\nu\left(I_{3}\right)$ and $\nu(I)<\nu\left(I_{6}\right)$, then the decorated graph has type IV.2 and the reduction type of the curve is Garden.

(v) If $\nu(I)=\nu\left(I_{3}\right)<\nu\left(I_{6}\right)$, then the decorated graph has type III.5, the reduction type of the curve is Tree, and the $j$-invariant of the genus-1 component of the special fiber is $j=2^{4}\left(I+2^{4} I_{3} I_{3}^{\prime}\right)^{3} /\left(I^{2} I_{3} I_{3}^{\prime}\right)$.

(vi) If $\nu(I)=\nu\left(I_{6}\right)<\nu\left(I_{3}\right)$, then the decorated graph has type III.6, the reduction type of the curve is Winky Cat, and the $j$-invariant of the genus-1 component of the special fiber is $j=2^{4}\left(I_{6}+2^{4} I\right)^{3} /\left(I_{6}^{2} I\right)$.

Proof. The conditions on the invariants imply that we may assume

$$
\nu\left(C \Delta_{c}\right)=0, \quad \nu(A)>0, \text { and } \nu\left(B \Delta_{b}\right)>0
$$

after permuting the variables, if necessary. In order to determine the stable reduction of $Y$ in the different subcases, we use two different coordinates for $X$. The coordinates

$$
\xi_{1}=\frac{\beta u+\alpha v+2 C w}{2(\alpha-a) v} \quad \xi_{2}=\frac{\beta u+\alpha v+2 C w}{2(\beta-b) u},
$$

correspond to $t_{1}=\left(P_{a}^{\prime}, P_{a}, P_{b}^{\prime}\right)$ and $t_{2}=\left(P_{b}, P_{b}^{\prime}, P_{a}\right)$ in the notation of Section 2.4. The coordinates $\xi_{1}$ and $\xi_{2}$ define models $\mathcal{X}_{1}$ and $\mathcal{X}_{2}$ of $X$, which may or may not be isomorphic over $\mathcal{O}$. We write $\bar{X}_{1}$ and $\bar{X}_{2}$ for the special fibers of the corresponding models. We use the same notation for further coordinates we introduce in the course of the proof. The coordinate $\xi_{1}$ is the same we considered in Example 2.13, which corresponds to case (vi) of the current lemma.

In this proof, we choose $\alpha$ to be a root of $p_{a}(T)=T^{2}-2 a T+4 B C$ of minimal valuation. Similarly, we choose $\beta$ and $\gamma$ to be a root of $p_{b}(T)=T^{2}-2 b T+4 A C$ and $p_{c}(T)=T^{2}-2 c T+4 A B$ of minimal valuation.

(i) If $2 \nu(I)>\nu\left(I_{3}\right)+\nu\left(I_{6}\right)>2 \nu\left(I_{3}\right)$ or $\nu\left(I_{3}\right)<\nu(I)<\nu\left(I_{6}\right)$, then $\nu\left(\Delta_{a}\right)>\nu(B)$ and $\nu\left(\Delta_{b}\right)>\nu(A)$. Moreover, $\nu\left(A \Delta_{a}\right)=\nu\left(B \Delta_{b}\right)$.

It is no restriction to assume that $\nu(B) \leq \nu(A)$. It follows that $2 \nu(\beta)=\nu(A) \geq$ $\nu(B)=2 \nu(\alpha)=2 \nu(a)$ and $2 \nu(\alpha-a)=\nu\left(\Delta_{a}\right)$.

We define a new coordinate

$$
\xi_{3}=\frac{\beta \tilde{\pi}_{3} u+\alpha \pi_{3} v+2 C \pi_{3} w}{2(\alpha-a) v},
$$

where $\pi_{3}$ and $\tilde{\pi}_{3}$ are chosen such that $\nu\left(-\beta B \tilde{\pi}_{3}+\alpha \gamma \pi_{3}\right)=\nu(\alpha-a)$. (This is obviously possible.) One computes that the points $P_{a}$ and $P_{a}^{\prime}$ both specialize to $\xi_{3}=0$ on $\bar{X}_{3}$, the points $P_{b}, P_{b}^{\prime}$, and $P_{c}$ specialize to $\xi_{3}=\infty$ on $\bar{X}_{3}$, and $\xi_{3}\left(P_{c}^{\prime}\right)$ specializes to a point with $\xi_{3} \neq 0, \infty$ on $\bar{X}_{3}$. 
Similarly, we find a coordinate $\xi_{4}$ such that the points $P_{a}, P_{a}^{\prime}$, and $P_{c}^{\prime}$ specialize to $\xi_{4}=0$ on $\bar{X}_{4}$, the points $P_{b}$ and $P_{b}^{\prime}$ specialize to $\xi_{4}=\infty$ on $\bar{X}_{4}$, and $\xi_{3}\left(P_{c}\right)$ specializes to a point with $\xi_{4} \neq 0, \infty$ on $\bar{X}_{4}$. We conclude that the decorated graph is of type IV.1, and the irreducible components of $\bar{X}$ are $\bar{X}_{1}, \bar{X}_{3}, \bar{X}_{4}, \bar{X}_{2}$ from left to right. The reduction type of the curve is Grl Pwr.

(ii) If $2 \nu(I)>\nu\left(I_{3}\right)+\nu\left(I_{6}\right)>2 \nu\left(I_{6}\right)$ or $\nu\left(I_{3}\right)>\nu(I)>\nu\left(I_{6}\right)$, then $\nu\left(\Delta_{a}\right)<\nu(B)$ and $\nu\left(\Delta_{b}\right)<\nu(A)$. We may assume that $\nu\left(\Delta_{b}\right) \leq \nu\left(\Delta_{a}\right)$.

One calculates that the points $P_{a}$ and $P_{c}^{\prime}$ both specialize to the point $\xi_{1}=1$, the points $P_{b}, P_{b}^{\prime}$, and $P_{c}$ specialize to the point $\xi_{1}=\infty$, and $P_{a}^{\prime}$ specializes to $\xi_{1}=0$ on $\bar{X}_{1}$. Similarly, one computes that the points $P_{a}, P_{a}^{\prime}, P_{c}^{\prime}$ specialize to the point $\xi_{2}=\infty$, the points $P_{b}^{\prime}$ and $P_{c}$ to the point $\xi_{2}=1$, and $P_{b}$ to $\xi_{2}=0$ on $\bar{X}_{2}$. We conclude that the decorated graph has type IV.3: the irreducible components $\bar{X}_{1}$ and $\bar{X}_{2}$ are the two middle components. The reduction type of the curve is Cat.

(iii) If $2 \nu(I) \geq \nu\left(I_{3}\right)+\nu\left(I_{6}\right)=2 \nu\left(I_{3}\right)$, then $\nu\left(\Delta_{a}\right)=\nu(B)$ and $\nu\left(\Delta_{b}\right)=\nu(A)$. It is no restriction to assume that $\nu(B) \leq \nu(A)$.

One computes that $P_{b}, P_{b}^{\prime}$, and $P_{c}$ all specialize to the point $\xi_{1}=\infty$ on $\bar{X}_{1}$. Moreover, the point $P_{c}^{\prime}$ specializes to a point with $\xi_{1} \neq 0,1, \infty$ on $\bar{X}_{1}$. In particular, the points $P_{a}, P_{a}^{\prime}, P_{c}^{\prime}$ and $P_{b}^{\prime}$ specialize to pairwise distinct points of $\bar{X}_{1}$. Similarly, one checks that the points $P_{b}, P_{b}^{\prime}, P_{c}$, and $P_{a}$ specialize to pairwise distinct points on $\bar{X}_{2}$. Hence $\bar{X}$ has type II.1 and the reduction type of the curve is Candy.

The stable reduction $\bar{Y}$ of $Y$ consists of two genus-1 curves intersecting in two points. To calculate their $j$-invariants we proceed as in Example 2.13. Let $\bar{Y}_{i}$ be the irreducible component of $\bar{Y}$ above $\bar{X}_{i}$ for $i=1,2$. The coordinate $\xi_{1}$ of $\bar{X}_{1}$ is identical to the coordinate from Example 2.13 , hence we find the same expression

$$
j\left(\bar{Y}_{1}\right) \equiv \frac{2^{6}\left(a^{2}+12 B C\right)^{3}}{\Delta_{a}^{2} \cdot 4 B C}(\bmod \pi) .
$$

A similar calculation yields

$$
j\left(\bar{Y}_{2}\right) \equiv \frac{2^{6}\left(b^{2}+12 A C\right)^{3}}{\Delta_{b}^{2} \cdot 4 A C} \quad(\bmod \pi) .
$$

One checks that the $j$-invariants $j\left(\bar{Y}_{1}\right)$ and $j\left(\bar{Y}_{2}\right)$ are the roots of the polynomial given in the statement.

(iv) Assume $\nu(I)<\nu\left(I_{3}\right)$ and $\nu(I)<\nu\left(I_{6}\right)$. It is no restriction to assume that $\nu\left(\Delta_{b}\right)<\nu(A)$. It follows that $\nu(B)<\nu\left(\Delta_{a}\right)$.

One computes that $P_{a}, P_{a}^{\prime}$, and $P_{c}^{\prime}$ specialize to the point $\xi_{2}=\infty$ and $P_{b}^{\prime}$ and $P_{c}$ specialize to the point $\xi_{2}=1$ on $\bar{X}_{2}$. By definition of $\xi_{2}$ the point $P_{b}$ specializes to $\xi_{2}=0$.

Define

$$
\xi_{3}=\frac{\gamma(1-2 B) u+4 B^{2} v+2 \alpha B w}{-2 B v} .
$$

Then the points $P_{a}$ and $P_{a}^{\prime}$ specialize to $\xi_{3}=0$, the points $P_{b}, P_{b}^{\prime}$, and $P_{c}$ specialize to $\xi_{3}=\infty$, and $P_{c}^{\prime}$ specializes to $\xi_{3}=1$ on $\bar{X}_{3}$. We conclude that the decorated graph has type IV.2. The components are $\bar{X}_{1}, \bar{X}_{3}, \bar{X}_{2}$, and a fourth one that we did not give a name. The reduction type of the curve is Garden.

(v) Assume $\nu(I)=\nu\left(I_{3}\right)<\nu\left(I_{6}\right)$. It is no restriction to assume that $\nu\left(\Delta_{b}\right)=\nu(A)$. It follows that $\nu(B)<\nu\left(\Delta_{a}\right)$. 
Case 1: We first consider the case that $\nu(A) \leq \nu(B)$.

Lemma 4.1 implies that $P_{a}, P_{a}^{\prime}$, and $P_{c}^{\prime}$ specialize to the same point $(0: 1: 0)$ of $\bar{X}_{0}$. The points $P_{b}$ and $P_{b}^{\prime}$ specialize to the same point of $\bar{X}_{0}$, as well, and that $P_{a}, P_{c}$, and $P_{b}$ specialize to pairside distinct points of $\bar{X}_{0}$. As in the previous cases, we may check that $\xi_{1}\left(P_{c}^{\prime}\right) \not \equiv 0,1, \infty(\bmod \pi)$. We conclude that the decorated graph has type III.5. The coordinates are $\bar{X}_{1}, \bar{X}_{0}$, and $\bar{X}_{2}$ from left to right.

The reduction type of $\bar{Y}$ is Tree. The genus- 1 component $\bar{Y}_{1}$ of the stable reduction is the normalization of the component corresponding to coordinate $\xi_{1}$. Again, we are in the situation of Example 2.13 and get

$$
j\left(\bar{Y}_{1}\right) \equiv \frac{2^{6}\left(a^{2}+12 B C\right)^{3}}{\Delta_{a}^{2} \cdot 4 B C} \quad(\bmod \pi) .
$$

In terms of invariants this can be expressed as

$$
j\left(\bar{Y}_{1}\right) \equiv 2^{4}\left(I+2^{4} I_{3} I_{3}^{\prime}\right)^{3} /\left(I^{2} I_{3} I_{3}^{\prime}\right) .
$$

Case 2: If we are not in case 1 than $\nu(A)>\nu(B)$. In this case $P_{b}, P_{b}^{\prime}$, and $P_{c}$ (resp. $\left.P_{a}, P_{a}^{\prime}\right)$ specialize to the same point of $\bar{X}_{0}$, and $P_{a}, P_{b}, P_{c}^{\prime}$ specialize to pairwise distinct points on $\bar{X}_{0}$. Moreover, $\overline{\xi_{2}\left(P_{c}^{\prime}\right) \not \equiv 0,1, \infty}$.

As in the previous case, the decorated graph has type III.5 and the reduction type of $\bar{Y}$ is tree. The component of genus 1 is the unique irreducible component $\bar{Y}_{2}$ above the component $\bar{X}_{2}$ corresponding to the coordinate $\xi_{2}$. We get

$$
j\left(\bar{Y}_{2}\right) \equiv \frac{2^{6}\left(b^{2}+12 A C\right)^{3}}{\Delta_{b}^{2} \cdot 4 A C} \quad(\bmod \pi) .
$$

In terms of invariants we get the same expression as in the above case,

$$
j\left(\bar{Y}_{2}\right) \equiv 2^{4}\left(I+2^{4} I_{3} I_{3}^{\prime}\right)^{3} /\left(I^{2} I_{3} I_{3}^{\prime}\right) .
$$

(vi) Assume that $\nu(I)=\nu\left(I_{6}\right)<\nu\left(I_{3}\right)$. It is no restriction to assume that $\nu\left(\Delta_{b}\right)<$ $\nu(A)$ and $\nu(B)=\nu\left(\Delta_{a}\right)$.

One computes that the points $P_{a}, P_{a}^{\prime}, P_{b}^{\prime}$, and $P_{c}^{\prime}$ specialize to pairwise distinct points on $\bar{X}_{1}$. Moreover, the points $P_{b}, P_{b}^{\prime}$ and $P_{c}$ specialize to the same point of $\bar{X}_{1}$.

The points $P_{a}, P_{a}^{\prime}$, and $P_{c}^{\prime}$ specialize to the same point $\xi_{1}=\infty$, the points $P_{b}^{\prime}$ and $P_{c}$ specialize to the point $\xi_{2}=1$, and $P_{b}$ specializes to the point $\xi_{2}=0$ on $\bar{X}_{2}$. This shows that $\bar{X}$ has type III.6: the irreducible components are $\bar{X}_{1}, \bar{X}_{2}$, and a further component from left to right. The reduction type of the curve $Y$ is Winky Cat.

As in Example 2.13 one computes that the $j$-invariant of the irreducible component $\bar{Y}_{1}$ above $\bar{X}_{1}$ is

$$
j\left(\bar{Y}_{1}\right) \equiv \frac{2^{6}\left(a^{2}+12 B C\right)^{3}}{\Delta_{a}^{2} \cdot 4 B C} \quad(\bmod \pi) .
$$

In terms of invariants, this is

$$
j\left(\bar{Y}_{1}\right) \equiv 2^{4}\left(I_{6}+2^{4} I\right)^{3} /\left(I_{6}^{2} I\right) .
$$

Lemma 4.7 (Theorem 3.7, case $(\mathrm{g})$ ). Let $Y$ be as in Theorem 3.7, in particular $\nu\left(I_{3}^{\prime \prime}\right)=0$. Assume that $\nu\left(I_{3}\right)>0, \nu\left(I_{3}^{\prime}\right)=0, \nu\left(I_{6}\right)=0$ and $\nu(I)>0$. Then the decorated graph has type III.3 and the reduction type of the curve is Loop. The j-invariant of the genus-1 
component is

$$
j=16 \frac{\left(I_{3}^{\prime \prime 2}-16 I_{3}^{\prime} I_{3}^{\prime \prime}+16 I_{3}^{\prime 2}\right)^{3}}{I_{3}^{\prime} I_{3}^{\prime \prime 4}\left(I_{3}^{\prime}-I_{3}^{\prime \prime}\right)} .
$$

Proof. From the conditions on the invariants, it follows that $\nu\left(\Delta_{a}\right)=\nu\left(\Delta_{b}\right)=\nu\left(\Delta_{c}\right)=0$ and exactly two among $\nu(A), \nu(B), \nu(C)$ are positive. Now Lemma 4.1 implies that branch points with the same inertia generator do not specialize to the same point on $\bar{X}_{0}$. Without loss of generality, we may assume that $A$ and $B$ have positive valuation and that $\nu(C)=0$. We conclude that the points $P_{a}, P_{a}^{\prime}, P_{b}, P_{b}^{\prime}$ specialize to pairwise distinct points of $\bar{X}_{0}$.

Up to possibly interchanging $P_{c}$ and $P_{c}^{\prime}$, it follows from Lemma 4.1 that $P_{c}$ specializes to the same point as one of $\left\{P_{b}, P_{b}^{\prime}\right\}$ on $\bar{X}_{0}$. The same argument using that $\nu(B)>0$ implies that $P_{c}^{\prime}$ specializes to the same point as one of $\left\{P_{a}, P_{a}^{\prime}\right\}$ on $\bar{X}_{0}$.

Hence the decorated graph has type III.3: the irreducible component $\bar{X}_{0}$ is the middle component of $\bar{X}$. The reduction type of the curve is Loop.

Let $\bar{Y}_{0}$ be the irreducible component above $\bar{X}_{0}$ of the stable reduction $\bar{Y}$ of $Y$. To compute $j\left(\bar{Y}_{0}\right)$ we argue as in the proof of Lemma 4.2 . After applying a suitable coordinate change in $\mathrm{GL}_{3}(K)$ on $Y$, we find a birational equation for $\bar{Y}_{0}$ :

$$
\bar{Y}_{0}:\left(c x^{2}+a z^{2}\right) y^{2}+b x^{2} z^{2}+C z^{4}=0 .
$$

Setting $x=1$ we recognize $\bar{Y}_{0}$ as elliptic curve and find

$$
j\left(\bar{Y}_{0}\right)=\frac{16\left(a^{2} b^{2}+14 a b c C+c^{2} C^{2}\right)^{3}}{a b c C(a b-c C)^{4}} \equiv \frac{16\left(I_{3}^{\prime \prime 2}-16 I_{3}^{\prime} I_{3}^{\prime \prime}+16 I_{3}^{\prime 2}\right)^{3}}{I_{3}^{\prime} I_{3}^{\prime \prime 4}\left(I_{3}^{\prime}-I_{3}^{\prime \prime}\right)} \quad(\bmod \pi) .
$$

Lemma 4.8 (Theorem 3.7, case $(\mathrm{h})$ ). Let $Y$ be as in Theorem 3.7, in particular $\nu\left(I_{3}^{\prime \prime}\right)=0$. Assume $\nu\left(I_{3}\right)>0, \nu\left(I_{3}^{\prime}\right)>0, \nu(I)>0$, and $\nu\left(I_{6}\right)=0$. Then the decorated graph has type $I V^{*} .3$ and the reduction type of the curve is Looop.

Proof. From the conditions on the invariants, it follows that $\nu\left(\Delta_{a}\right)=\nu\left(\Delta_{b}\right)=\nu\left(\Delta_{c}\right)=0$ and $\nu(A), \nu(B), \nu(C)$ are positive. Lemma 4.1 implies that branch points with the same inertia generator do not specialize to the same point on $\bar{X}_{0}$. Moreover, for every pair $i \neq$ $j \in\{a, b, c\}$ one of the branch points with inertia generator $\sigma_{i}$ and one of the branch points with inertia generator $\sigma_{j}$ specialize to the same point of $\bar{X}_{0}$. We conclude that the decorated graph has type $\mathrm{IV}^{*} .3$.

4.2. Main result with degenerate conic. As in Section 4.1 we write $\mathcal{X}_{0}$ for the model of $X$ defined by $(2.2)$ and $\bar{X}_{0}$ for its special fiber. Since we assume that the left-hand side of the equation (2.2) for $X$ is normalized as in Proposition $3.1 \mathcal{X}_{0}$ is indeed a model, and $\bar{X}_{0}$ is a conic over the residue field $k$ of $K$. In this section we prove Theorem 3.8 , which treats the case that $\bar{X}_{0}$ is degenerate. Recall that this implies that $I_{3}^{\prime \prime}=\Delta(X)$ has positive valuation. The classification of degenerate conics in characteristic different from 2 implies therefore that $\bar{X}_{0}$ is either reducible or non-reduced. In the first case, $\bar{X}_{0}$ consist of two irreducible components. In the second case the underlying reduced scheme $\bar{X}_{0}^{\text {red }}$ is irreducible.

Proposition 4.9. Assume that $\nu\left(I_{3}^{\prime \prime}\right)$ is positive.

(i) The curve $\bar{X}_{0}$ is reducible and the points $P_{a}, P_{a}^{\prime}$ specialize to different irreducible components of $\bar{X}_{0}$ if and only if $\nu\left(\Delta_{a}\right)$ is zero. 
(ii) The curve $\bar{X}_{0}$ is non-reduced and $\bar{X}_{0}^{\mathrm{red}}$ is irreducible if and only if $\nu\left(\Delta_{a}\right), \nu\left(\Delta_{b}\right)$ and $\nu\left(\Delta_{c}\right)$ are all positive.

(iii) Assume that $\bar{X}_{0}$ is reduced and $\nu(C)=0$. Let $\alpha$ (respectively $\beta$ ) be a root of $p_{a}(T)=T^{2}-2 a T+4 B C$ (respectively $\left.p_{b}(T)=T^{2}-2 b T+4 A C\right)$. Then the two points $P_{a}^{\prime}=(0:-2 C: \alpha)$ and $P_{b}=(-2 C: 0: \beta)$ specialize to the same irreducible component of $\bar{X}_{0}$ if and only if the valuation of $\alpha(2 b-\beta)+(2 a-\alpha) \beta-4 C c$ is positive.

Proof. (i) If $\nu\left(\Delta_{a}\right)$ is positive, then the two roots of $T^{2}-2 a T+4 B C$ are congruent modulo $\pi$, thus $P_{a}, P_{a}^{\prime}$ specialize to the same point on $\bar{X}_{0}$.

Assume that $\nu\left(\Delta_{a}\right)=0$ and that $P_{a}, P_{a}^{\prime}$ specialize to the same irreducible component of $\bar{X}_{0}$. This also includes the case that $\bar{X}_{0}^{\text {red }}$ is reduced. We denote the irreducible component of $\bar{X}_{0}^{\text {red }}$ to which $P_{a}, P_{a}^{\prime}$ specialize by $\bar{X}_{1}$. It follows from $(2.2)$ that $u=0$ is an equation for $\bar{X}_{1}$. But $u$ is a factor of the left-hand side of $(2.2)(\bmod \pi)$ if and only if the valuations of $B, C$, and $a$ are positive, which contradicts the assumption $\nu\left(\Delta_{a}\right)=0$. Statement (i) follows.

(ii) Assume that $\bar{X}_{0}$ is non-reduced. Then $\bar{X}_{0}^{\text {red }}$ is irreducible and the left-hand side of (2.2) modulo $\pi$ is a square. We conclude that we may choose square roots of $A, B, C(\bmod \pi)$ such that

$$
2 \sqrt{A B} \equiv c, \quad 2 \sqrt{A C} \equiv b, \quad 2 \sqrt{B C} \equiv a .
$$

This implies that that valuations of $\Delta_{a}, \Delta_{b}$ and $\Delta_{c}$ are positive. The converse is similar. Statement (ii) follows.

(iii) Assume that $\bar{X}_{0}$ is reduced and $\nu(C)=0$. We write $\bar{X}_{1}$ and $\bar{X}_{2}$ for the irreducible components of $\bar{X}_{0}$.

Note that if $P_{a}^{\prime}$ and $P_{b}$ specialize to the same irreducible component $\bar{X}_{1}$ of $\bar{X}_{0}$, then $\bar{X}_{1}$ is defined by

$$
\bar{X}_{1}: \beta u+\alpha v+2 C w=0 .
$$

We write $\alpha^{\prime}=2 a-\alpha$ (resp. $\left.\beta^{\prime}=2 b-\beta\right)$ for the second root of $p_{a}$ (resp. $p_{b}$ ). Statement (i) implies that the points $P_{a}=(0: \alpha:-2 B)=\left(0:-2 C: \alpha^{\prime}\right)$ and $P_{b}^{\prime}=(\beta: 0:-2 A)=\left(-2 C: 0: \beta^{\prime}\right)$ specialize to $\bar{X}_{2}$, which may be given by

$$
\bar{X}_{2}: \beta^{\prime} u+\alpha^{\prime} v+2 C w=0 .
$$

Computing the product of the equations for $\bar{X}_{1}$ and $\bar{X}_{2}$ we obtain

$$
\begin{gathered}
(\beta u+\alpha v+2 C w)\left(\beta^{\prime} u+\alpha^{\prime} v+2 C w\right)= \\
4 C A u^{2}+4 B C v^{2}+4 C^{2} w^{2}+4 C a v w+4 C b u w+\left(\alpha \beta^{\prime}+\alpha^{\prime} \beta\right) u v .
\end{gathered}
$$

The right-hand side of this equation is congruent to the left-hand side of $(2.2)$ if and only if

$$
\nu(\alpha(2 b-\beta)+(2 a-\alpha) \beta-4 C c)>0 .
$$

Lemma 4.10 (Theorem 3.8, case (a)). Let $Y$ be as in Theorem 3.8, in particular we have $\nu\left(I_{3}^{\prime \prime}\right)>0$. Assume that $\nu\left(I_{3}\right)=\nu\left(I_{6}\right)=0$. Then the decorated graph has type II.2 and the reduction of the curve is $D N A$.

Proof. From the conditions on the invariants, it follows that the valuations of $A, B, C, a, b, c$ are zero. Then, by Lemma 4.1, all 6 branch points specialize to pairwise distinct points of $\bar{X}_{0}$. Proposition 4.9 implies that $\bar{X}_{0}$ reducible, and the branch points with the same 
inertia generator specialize to different irreducible components of $\bar{X}_{0}$. Therefore the decorated graph has type II.2 thus the reduction of the curve is DNA.

Lemma 4.11 (Theorem 3.8, case (b)). Let $Y$ be as in Theorem 3.8, in particular $\nu\left(I_{3}^{\prime \prime}\right)>$ 0 . Assume that $\nu\left(I_{3}+I_{3}^{\prime}\right)=0, \nu\left(I_{6}\right)>0$ and $\nu(I)=0$. Then one of the following occurs:

(i) if $\nu\left(I_{6}\right)>\nu\left(I_{3}^{\prime \prime}\right)$, the decorated graph has type $I V^{*}$.2 and the reduction of the curve is $D N A$;

(ii) if $\nu\left(I_{6}\right)<\nu\left(I_{3}^{\prime \prime}\right)$, the decorated graph has type $I V .5$ and the reduction of the curve is Braid; and

(iii) otherwise, the decorated graph has type III.4, the reduction type of the curve is Candy, and the $j$-invariants of the genus-1 components of the special fiber are $j_{1}=j_{2}=1728$.

Proof. It follows from the conditions $\nu\left(I_{6}\right)>0$ and $\nu(I)=0$ that exactly one among $\nu\left(\Delta_{a}\right), \nu\left(\Delta_{b}\right), \nu\left(\Delta_{c}\right)$ is positive. Without loss of generality we may assume that $\nu\left(\Delta_{a}\right)>0$. Then $\nu(I)=0$ also implies $\nu(B C)=0$. It follows from Proposition 4.9 that $\bar{X}_{0}$ is reduced, has two irreducible components and the points $P_{a}, P_{a}^{\prime}$ both specialize to the intersection of these two components.

Now, fix $\beta$ and $\gamma$ to be roots of $p_{b}(T)=T^{2}-2 b T+4 A C$ and $p_{c}(T)=T^{2}-2 c T+4 A B$, respectively, that have valuation 0 .

Claim: We may choose $\beta$ and $\gamma$ so that they satisfy additionally that $\nu((2 b-\beta) \gamma+$ $\beta(2 c-\gamma)-4 A a)>0$. Moreover, there exists coordinates $\xi_{1}$ and $\xi_{2}$ such that $P_{a}$ and $P_{a}^{\prime}$ specialize to the intersection point $\tau$ of the corresponding irreducible components $\bar{X}_{1}$ and $\bar{X}_{2}$ and $P_{b}^{\prime}$ and $P_{c}$ (resp. $P_{b}$ and $P_{c}^{\prime}$ ) specialize to pairwise distinct points of $\bar{X}_{1}$ (resp. $\bar{X}_{2}$ ) different from $\tau$.

Assume first that $\nu(A)=0$. Then all the roots of $p_{b}$ and $p_{c}$ have valuation 0 . In this case it follows from the proof of Proposition 4.9. (iii) that we may choose $\beta$ and $\gamma$ as in the claim. That proof then also implies that $P_{b}^{\prime}$ and $P_{c}$ (resp. $P_{b}, P_{c}^{\prime}$ ) specialize to the same component of $\bar{X}_{0}$. Moreover, Proposition 4.9.(i) implies that the points $P_{b}, P_{b}^{\prime}, P_{c}, P_{c}^{\prime}$ specialize to pairwise distinct points on $\bar{X}_{0}$. This proofs the claim in this case.

Next assume that $\nu(A)$ is positive. Then $\nu((2 b-\beta) \gamma+\beta(2 c-\gamma)-4 A a)>0$ for any choice of $\beta$ and $\gamma$, but there are unique roots $\beta$ of $p_{b}$ and $\gamma$ of $p_{c}$ with $\nu(\beta)=\nu(\gamma)=0$. With this choice, we have that $P_{b}^{\prime}$ and $P_{c}$ both specialize to the point $(1: 0: 0)$ in the smooth locus of $\bar{X}_{0}$ and in particular to the same irreducible component of $\bar{X}$. Let $\xi_{1}$ and $\xi_{2}$ be coordinates corresponding to $t_{1}=\left(P_{b}^{\prime}, P_{c}, P_{a}\right)$ and $t_{2}=\left(P_{b}, P_{c}^{\prime}, P_{a}\right)$ as in Section 2.4. The corresponding components $\bar{X}_{1}$ and $\bar{X}_{2}$ satisfy the conditions in the claim.

Remains to compute a component separating $P_{a}, P_{a}^{\prime}$. Consider

$$
\xi_{3}=\frac{\beta u+\alpha v+2 C w}{2 v(\alpha-a)} .
$$

This is the coordinate corresponding to $t_{3}=\left(P_{a}^{\prime}, P_{a}, P_{b}^{\prime}\right)$. One checks that $\overline{\xi_{3}\left(P_{c}\right)}=\infty$. The decorated graph of $X$ depends on the value of $\overline{\xi_{3}\left(P_{b}\right)}=\overline{\xi_{3}\left(P_{c}^{\prime}\right)}$. Using $(2.2)$ and that $\beta$ is a root of $p_{b}$ we find the equivalent expression for the coordinate

$$
\xi_{3}=\frac{-2 \beta(c u+B v+a w)+\alpha(2 A u+\beta w)}{2(\alpha-a)(2 A u+\beta w)} .
$$

We obtain

$$
\xi_{3}\left(P_{b}\right)=\frac{4 c C-\alpha(2 b-\beta)-(2 a-\alpha) \beta}{4(\alpha-a)(\beta-b)} .
$$


Note that the discriminant factors as follows:

$$
\Delta(X)=(4 c C-\alpha(2 b-\beta)-(2 a-\alpha) \beta)(4 c C-\alpha \beta-(2 a-\alpha)(2 b-\beta)) .
$$

Let $\delta=\nu(-4 C c+\alpha(2 b-\beta)+(2 a-\alpha) \beta)$ and note

$$
\begin{aligned}
\nu(-4 C c+\alpha \beta+(2 a-\alpha)(2 b-\beta)) & =\nu(4(\alpha-a)(\beta-b)-4 C c+\alpha(2 b-\beta)+(2 a-\alpha) \beta) \\
& \geq \min (\nu((\alpha-a)(\beta-b)), \delta),
\end{aligned}
$$

where $\nu(\alpha-a)=\frac{1}{2} \nu\left(\Delta_{a}\right)$. Then, we are in one of the following cases:

- If $2 \delta<\nu\left(\Delta_{a}\right)$, then one has $\nu(\Delta(X))=2 \delta<\nu\left(\Delta_{a}\right)$ and $\overline{\xi_{3}\left(P_{b}\right)}=\infty$. In this case, $\bar{X}$ is of type $\mathrm{IV}^{*} .2$. The component $\bar{X}_{3}$ is the left most vertical component.

- If $2 \delta>\nu\left(\Delta_{a}\right)$, then one has $\nu(\Delta(X))=\delta+1 / 2 \nu\left(\Delta_{a}\right)>\nu\left(\Delta_{a}\right)$, and $\overline{\xi\left(P_{b}\right)}=0$. In this case, $\bar{X}$ is of type IV.5. The component $\bar{X}_{3}$ is the one in the middle that intersects $\bar{X}_{1}$.

- If $2 \delta=\nu\left(\Delta_{a}\right)$ and $\nu(\alpha \beta+(2 a-\alpha)(2 b-\beta)-4 C c)>\delta$, then one has $\nu(\Delta(X))>$ $\nu\left(\Delta_{a}\right)$, and $\overline{\xi\left(P_{b}\right)}=1$. In this case, $\bar{X}$ is again of type IV.5, but the component $\bar{X}_{3}$ is the one in the middle that intersects $\bar{X}_{2}$.

- Lastly, if $2 \delta=\nu\left(\Delta_{a}\right)$ and $\nu(\alpha \beta+(2 a-\alpha)(2 b-\beta)-4 C c)=\delta$, then one has $\nu(\Delta(X))=\nu\left(\Delta_{a}\right)$, and $\overline{\xi\left(P_{b}\right)} \neq 0,1, \infty$. In this case, $\bar{X}$ is of type III.4. The component $\bar{X}_{3}$ is the central one.

This yields the case distinction from the statement of the lemma.

It remains to compute the $j$-invariant of the two components of the stable reduction $\bar{Y}$ of $Y$ in case (iii) of the lemma. In this case $\bar{Y}$ consists of two genus-1 curves $\bar{Y}_{1}$ and $\bar{Y}_{2}$ intersecting in two points. These two curves are permuted by the action of $\sigma_{b}$ and $\sigma_{c}$. Hence $\bar{Y}_{1} \simeq \bar{Y}_{2}$. The map $f: Y \rightarrow X$ extends to a finite and flat map $\bar{f}: \bar{Y} \rightarrow \bar{X}_{3}$, where $\bar{X}_{3}$ is the special fiber of the model of $X$ defined by the coordinate $\xi_{3}$. Moreover, $\bar{f}$ is Galois with Galois group $V$.

The map $\bar{f}: \bar{Y} \rightarrow \bar{X}$ factors as $\bar{Y} \rightarrow \bar{Y} /\left\langle\sigma_{i}\right\rangle \rightarrow \bar{X}$ for $i \in\{a, b, c\}$. We write $\bar{Y}^{\circ}=\bar{Y}_{1} \sqcup \bar{Y}_{2}$ for the normalization of $\bar{Y}$. The induced map $\bar{f}: \bar{Y}^{\circ} \rightarrow \bar{Y} /\left\langle\sigma_{i}\right\rangle$ for $i=b, c$ just identified the two irreducible components. It follows that the elliptic curve $E_{i}=Y /\left\langle\sigma_{i}\right\rangle$ has good reduction for $i=b, c$ and its reduction is $\overline{E_{i}}=\bar{Y} /\left\langle\sigma_{i}\right\rangle$. This implies that $\overline{E_{b}}$ and $\overline{E_{c}}$ are elliptic curves and they are both isomorphic to $\bar{Y}_{1} \simeq \bar{Y}_{2}$. Therefore

$$
j\left(\bar{Y}_{1}\right) \equiv j\left(\bar{Y}_{2}\right) \equiv j\left(E_{b}\right) \equiv j\left(E_{c}\right) \quad(\bmod \pi) .
$$

To compute $j\left(\bar{Y}_{1}\right)$ it therefore to compute the reduction of $j\left(E_{b}\right)(\bmod \pi)$.

We have $E_{b}: A x^{4}+B v^{2}+C z^{4}+a v z^{2}+b z^{2} x^{2}+c x^{2} v=0$. After a change of coordinates in $\mathrm{GL}_{3}(\mathcal{O})$, we can assume $B=C=1, a=2+a_{1} \pi_{1}$ and $c=b+b_{1} \pi_{1}^{2}$ for a suitable element $\pi_{1} \in \mathcal{O}$ of positive valuation. A direct computation of the $j$-invariant yields $j\left(E_{b}\right) \equiv 1728 \bmod \pi$.

Lemma 4.12 (Theorem 3.8, case (c)). Let $Y$ be as in Theorem 3.8 and assume that $\nu\left(I_{3}\right)=0, \nu\left(I_{3}^{\prime}\right)>0, \nu\left(I_{6}\right)>0$ and $\nu(I)>0$. Then $Y$ has (maybe bad) hyperelliptic reduction. More specifically,

(i) if $\nu\left(I_{6}^{2}\right)=\nu\left(I_{3} I_{3}^{\prime \prime 3}\right), \nu\left(I_{3}^{\prime 4}\right) \geq \nu\left(I_{3} I_{3}^{\prime \prime}\right)$, then $Y$ has good hyperelliptic reduction,

(ii) if $\nu\left(I_{6}^{2}\right)>\nu\left(I_{3} I_{3}^{\prime \prime 3}\right), \nu\left(I_{3}^{\prime 4}\right) \geq \nu\left(I_{3} I_{3}^{\prime \prime}\right)$, and $\nu\left(I_{3}^{\prime 4}-4 I_{3} I_{3}^{\prime \prime}\right)=\nu\left(I_{3} I_{3}^{\prime \prime}\right)$, then the decorated graph has type II.3 and the reduction of the curve is Loop,

(iii) if $\nu\left(I_{6}^{2}\right)>\nu\left(I_{3} I_{3}^{\prime \prime 3}\right), \nu\left(I_{3}^{\prime 4}\right)=\nu\left(I_{3} I_{3}^{\prime \prime}\right)$, and $\nu\left(I_{3}^{\prime 4}-4 I_{3} I_{3}^{\prime \prime}\right)>\nu\left(I_{3} I_{3}^{\prime \prime}\right)$, then the decorated graph has type III.1 and the reduction of the curve is DNA,

(iv) if $\nu\left(I_{6}^{2}\right)<\nu\left(I_{3} I_{3}^{\prime \prime 3}\right), \nu\left(I_{3}^{\prime 4}\right) \geq \nu\left(I_{3} I_{6}\right)$, then the decorated graph has type II.2 and the reduction of the curve is DNA, 
(v) if $12 \nu\left(I_{3}^{\prime}\right)<3 \nu\left(I_{3} I_{3}^{\prime \prime}\right)<2 \nu\left(I_{3} I_{6}\right)$, then the decorated graph has type $I V^{*} .2$ and the reduction of the curve is $D N A$,

(vi) if $12 \nu\left(I_{3}^{\prime}\right)<2 \nu\left(I_{3} I_{6}\right)<3 \nu\left(I_{3} I_{3}^{\prime \prime}\right)$, then the decorated graph has type $I V .5$ and the reduction of the curve is Braid, and

(vii) if $12 \nu\left(I_{3}^{\prime}\right)<2 \nu\left(I_{3} I_{6}\right)=3 \nu\left(I_{3} I_{3}^{\prime \prime}\right)$, then the decorated graph has type III.4 and the reduction of the curve is Candy.

Proof. It follows from the conditions on the invariants that $\nu(A), \nu(B)$ and $\nu(C)$ are zero and that $\nu\left(\Delta_{a}\right), \nu\left(\Delta_{b}\right)$ and $\nu\left(\Delta_{c}\right)$ are all positive.

Therefore, the special fiber $\bar{X}_{0}$ of the model of $X$ defined by 2.2 non-reduced, see Proposition 4.9. We claim that $Y$ has (not necessarily good) hyperelliptic reduction.

Note that there exist a choice of square roots such that the equation of $Y$ can be written as

$$
\left(\sqrt{A} x^{2}+\sqrt{B} y^{2}+\sqrt{C} z^{2}\right)+(a-2 \sqrt{B C}) y^{2} z^{2}+(b-2 \sqrt{A C}) x^{2} z^{2}+(c-\sqrt{A B}) x^{2} y^{2}=0,
$$

in such a way that the coefficients of $x^{2} y^{2}, y^{2} z^{2}$ and $z^{2} x^{2}$ have all positive valuation. Let $\pi_{1} \in \mathcal{O}$ be an element with valuation $\min \{\nu(a-2 \sqrt{B C}), \nu(b-2 \sqrt{A C}), \nu(c-\sqrt{A B})\} / 2$. Assume that the minimum of these valuations is attained by $a-2 \sqrt{B C}$, then we can rewrite the equation of $Y$ as follows:

$$
Y:\left\{\begin{array}{l}
\pi_{1}^{2} t^{2}=-\left((a-2 \sqrt{B C}) y^{2} z^{2}+(b-2 \sqrt{A C}) x^{2} z^{2}+\left(c-\sqrt{A B} x^{2} y^{2}\right)^{r}\right) \\
\pi_{1} t=\sqrt{A} x^{2}+\sqrt{B} y^{2}+\sqrt{C} z^{2}
\end{array} .\right.
$$

After making a suitable change of coordinates in $\mathrm{GL}_{3}(K)$ that sends the conic $\sqrt{A} x^{2}+$ $\sqrt{B} y^{2}+\sqrt{C} z^{2}=0$ to $x_{1}^{2}-y_{1} z_{1}=0$, and taking $z_{1}=1$ and $y_{1}=x_{1}^{2}$ in the reduction of the first equation defining $Y$ in 4.2 , we get a hyperelliptic equation $t^{2}=x_{1}^{8}+M x_{1}^{6}+$ $N x_{1}^{4}+M x_{1}^{2}+1$ with coefficients:

$$
\begin{gathered}
M=-4 \frac{b \sqrt{B}-c \sqrt{C}}{\sqrt{A}(a-2 \sqrt{B C})} \\
N=-2+8 \frac{b \sqrt{B}+c \sqrt{C}-4 \sqrt{A B C}}{\sqrt{A}(a-2 \sqrt{B C})} .
\end{gathered}
$$

Its invariants, as defined in Proposition 5.2 , are

$$
\left(L_{1}: L_{2}: L_{3}\right)=\left(2 I_{3}^{\prime}: 16 I_{3} I_{3}^{\prime \prime}:-4 I_{6} I_{3}\right) \in \mathbb{P}_{1,2,3}^{2} .
$$

Notice that this equality of projective points is not a coordinate-wise equality, but one in a weighted projective space.

Let $\pi_{2} \in \mathcal{O}$ be an element of valuation $\min \left(\nu\left(L_{1}\right), \nu\left(L_{2}\right) / 2, \nu\left(L_{3}\right) / 3\right)$. Then we have

$$
\left(L_{1}: L_{2}: L_{3}\right)=\left(\frac{2 I_{3}^{\prime}}{\pi_{2}}: \frac{16 I_{3} I_{3}^{\prime \prime}}{\pi_{2}^{2}}: \frac{-4 I_{6} I_{3}}{\pi_{2}^{3}}\right) \in \mathbb{P}_{1,2,3}^{2}
$$

This is still not a coordinate-wise equality, but a coordinate-wise valuation equality. It follows that we may assume that $\min \left(\nu\left(L_{1}\right), \nu\left(L_{2}\right), \nu\left(L_{3}\right)\right)=0$. Rewriting the normalized invariants in Theorem 5.6 in terms of $I_{3}, I_{3}^{\prime}, I_{3}^{\prime \prime}, I_{6}$ and $I$ yields the result.

Lemma 4.13 (Theorem 3.8, case (d)). Let $Y$ be as in Theorem 3.8, in particular $\nu\left(I_{3}^{\prime \prime}\right)>$ 0 . Assume that $\nu\left(I_{3}\right)>0, \nu\left(I_{6}\right)=0$ and $\nu(I)=0$, then the decorated graph has type III. 7 and the reduction type of the curve is Cave. 
Proof. From the conditions on the invariants, it follows that $\nu\left(\Delta_{a}\right)=\nu\left(\Delta_{b}\right)=\nu\left(\Delta_{c}\right)=0$ and exactly one of $\nu(A), \nu(B), \nu(C)$ is positive. We may assume that $\nu(A)>0$. Proposition 4.9 implies that $\bar{X}_{0}$ is reducible and that branch points with the same inertia generator specialize to different irreducible components of $\bar{X}_{0}$. Proposition 3.1 implies that one of branch points $P_{b}, P_{b}^{\prime}$ and one of the branch points $P_{c}, P_{c}^{\prime}$ specialize to the same irreducible component of $\bar{X}_{0}$. Moreover, that proposition implies that none of the branch points specialize to the singular point of $\bar{X}_{0}$. Hence the decorated graph has type III.7 and the reduction type of the curve is Cave.

Lemma 4.14 (Theorem 3.8, case (e)). Let $Y$ be as in Theorem 3.8, in particular $\nu\left(I_{3}^{\prime \prime}\right)>$ 0 . Assume that $\nu\left(I_{3}\right)>0, \nu\left(I_{3}^{\prime}\right)=0$ and $\nu(I)>0$, then the decorated graph has type $I V .4$ and the reduction of the curve is Braid.

Proof. From the conditions on the invariants and (3.1), it follows that $\nu\left(\Delta_{a}\right)=\nu\left(\Delta_{b}\right)=$ $\nu\left(\Delta_{c}\right)=0$ and exactly two among $\nu(A), \nu(B), \nu(C)$ are positive. It is no restriction to assume that $\nu(C)=0$. Now Proposition 4.9 implies that $\bar{X}_{0}$ is reducible and that branch points with the same inertia generator specialize to different irreducible components of $\bar{X}_{0}$. Moreover, Proposition 4.9. (iii) implies that $P_{c}$ specializes to the same point as one of $\left\{P_{b}, P_{b}^{\prime}\right\}$ on $\bar{X}$ and $P_{c}^{\prime}$ specializes to the same point as one of $\left\{P_{a}, P_{a}^{\prime}\right\}$ up to renaming $P_{c}$ and $P_{c}^{\prime}$. Hence the decorated graph has type IV.4. The two irreducible components of $\bar{X}_{0}$ are the central onces. The reduction of the curve is Braid.

\section{Hyperelliptic CASE}

In this section we give an analogous result to Theorems 3.7 and 3.8 for the hyperelliptic case, i.e., for curves in $\mathcal{M}_{3, V}^{\text {hyp }}$. Recall that $(K, \nu)$ is a complete discretely valued field of characteristic 0 and residue characteristic $2 \neq p \geq 0$. Recall that we replace $K$ by a finite extension, if necessary, without changing the notation.

Let $Y / K$ be a genus-3 hyperelliptic curve such that $\operatorname{Aut}_{\bar{K}}(Y)$ contains a subgroup $V \simeq C_{2} \times C_{2}$ such that for every non-trivial element $\sigma \in V$ the quotient $Y /\langle\sigma\rangle$ has genus 1. Then we can write (see [Bou98, Section 4.3] or [LR12, Table 3]):

$$
Y: y^{2}=x^{8}+M x^{6}+N x^{4}+M x^{2}+1,
$$

and we identify $V$ with the group generated by

$$
\sigma_{1}(x, y)=(-x, y) \text { and } \sigma_{2}(x, y)=\left(1 / x, y / x^{4}\right) .
$$

We set $\sigma_{3}:=\sigma_{1} \sigma_{2}$.

In particular, the genus-3 hyperelliptic curve $Y$ is the $V$-Galois cover of a conic, and we obtain the following diagram.

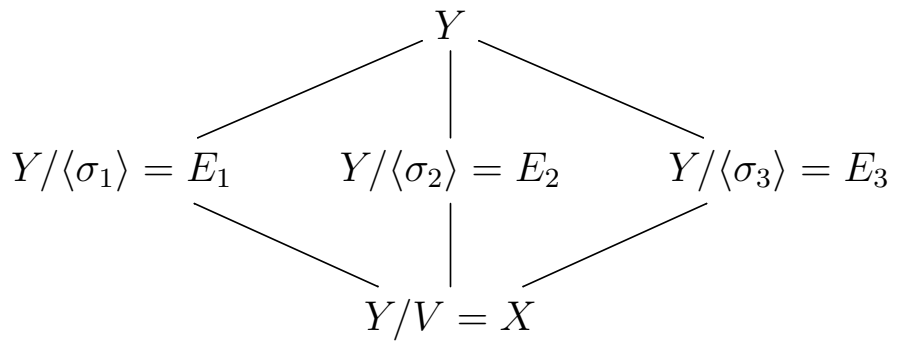

where

$$
\begin{aligned}
& Y: y^{2}=x^{8}+M x^{6}+N x^{4}+M x^{2}+1 \\
& X: w^{2}=v^{2}+(M-4) v+(-2 M+N+2)
\end{aligned}
$$


with $w=\frac{y}{x^{2}}$ and $v=\left(x+\frac{1}{x}\right)^{2}$. Moreover, we compute the discriminants

$$
\begin{aligned}
& \Delta(Y)=2^{4}(-2 M+N+2)^{2}(2 M+N+2)^{2}\left(M^{2}-4 N+8\right)^{4}, \\
& \Delta(X)=M^{2}-4 N+8 .
\end{aligned}
$$

The problem with the hyperelliptic model given in (5.1) is that it is singular at the infinity point $(0: 1: 0)$ and it is not easy to keep track of the ramification data.

Instead, we choose to work with the smooth model:

$$
Y:\left\{\begin{array}{l}
t^{2}=y^{4}+M y^{3} z+N y^{2} z^{2}+M y z^{3}+z^{4}, \quad \subseteq \mathbb{P}_{1,1,1,2}^{3} \\
0=x^{2}-y z
\end{array}\right.
$$

where now the automorphisms are given by

$$
\sigma_{1}((x: y: z: t))=(-x: y: z: t) \text { and } \sigma_{2}((x: y: z: t))=(x: z: y: t) .
$$

We get the following equation for the conic $X:=Y / V$ :

$$
\begin{aligned}
Y & \rightarrow X: w^{2}=v^{2}+(M-4) u v+(-2 M+N+2) u^{2} \subseteq \mathbb{P}^{2} \\
(x: y: z: t) & \mapsto(u: v: w)=\left(x^{2}:(y+z)^{2}: t\right),
\end{aligned}
$$

and the six branch points in $X$ are

$$
\begin{array}{ll}
\sigma_{1}: P_{1}=(0: 1: 1), & P_{1}^{\prime}=(0: 1:-1), \\
\sigma_{2}: P_{2}=(1: 4: \lambda), & P_{2}^{\prime}=(1: 4:-\lambda), \\
\sigma_{3}: P_{3}=(1: 0: \mu), & P_{3}^{\prime}=(1: 0:-\mu),
\end{array}
$$

where $\lambda$ is a root of $T^{2}-(2 M+N+2)=0$ and $\mu$ is a root of $T^{2}-(-2 M+N+2)=0$.

Note that the discriminant of this conic is still $\Delta(X)=M^{2}-4 N+8$.

Remark 5.1. If $\nu(\Delta(X))>0$, then the conic always reduces to the product of two different lines $\left(\frac{M-4}{2} u+v-w\right)\left(\frac{M-4}{2} u+v+w\right)$.

5.1. Invariants. Shioda [Shi67] gives 9 invariants $J_{2}, J_{3}, \ldots, J_{10}$ for genus-3 hyperelliptic curves, which we call the Shioda invariants. The Shioda invariants parametrize the 5dimensional locus of hyperelliptic curves $\mathcal{M}_{3}^{\text {hyp }}$ inside the moduli space of genus- 3 curves $\mathcal{M}_{3}$. The stratum $\mathcal{M}_{3, V}^{\text {hyp }}$ is the intersection of $\mathcal{M}_{3, V}$ with $\mathcal{M}_{3}^{\text {hyp }}$, and by Lemma 2.1. (3) it is a 2-dimensional stratum.

In [LR12, Lemma 3.14], the authors give (sufficient and necessarily) conditions in terms of the Shioda invariants for a curve to belong to the stratum $\mathcal{M}_{3, V}^{\text {hyp }}$. Loc. cit. also gives expressions to compute parameters $M, N$ from the Shioda invariants, thus obtaining a model

$$
Y: y^{2}=x^{8}+M x^{6}+N x^{4}+M x^{2}+1 .
$$

However, using the Shioda invariants restricted to the stratum $\mathcal{M}_{3, V}^{\text {hyp }}$ is not practical to characterize the stable reduction of $Y$, so we define invariants for the stratum $\mathcal{M}_{3, V}^{\text {hyp }}$.

Proposition 5.2. The invariant ring of $\mathcal{M}_{3, V}^{\text {hyp }}$ is generated by the following invariants of weight 1,2 and 3 respectively:

$$
L_{1}=N+10, L_{2}=M^{2}-4 N+8, L_{3}=(2 M+N+2)(2 M-N-2) .
$$

Proof. First we need to check that they are invariants. Secondly that they generate the ring of invariants.

Isomorphisms between hyperelliptic curves $y^{2}=f(x, z)$ are given by linear maps $(x, z) \mapsto\left(a_{11} x+a_{12} z, a_{21} x+a_{22} z\right)$. Since the isomorphisms between curves in the family $y^{2}=x^{8}+M x^{6}+N x^{4}+M x^{2}+1$ preserve the automorphism group generated by 
$(x, z) \mapsto(-x, z)$ and $(x, z) \mapsto(z, x)$, every isomorphism can be written as a composition of $i:(x, z) \mapsto(x,-z), r:(x, z) \mapsto(x+z, x-z)$ and automorphisms of the curve. Notice that $r^{2}=i^{2}=(r i)^{3}=\mathrm{Id}$, and that they generate a finite group $G$ isomorphic to $S_{3}$.

This implies that all the curves isomorphic to the one with parameters $(M, N)$ are the curves with parameters:

$$
\begin{gathered}
(M, N),(-M, N), \\
((8 M-4 N+56) /(2 M+N+2),(-20 M+6 N+140) /(2 M+N+2)) \\
(-(8 M-4 N+56) /(2 M+N+2),(-20 M+6 N+140) /(2 M+N+2)), \\
((-8 M-4 N+56) /(-2 M+N+2),(20 M+6 N+140) /(-2 M+N+2)), \\
(-(-8 M-4 N+56) /(-2 M+N+2),(20 M+6 N+140) /(-2 M+N+2)) .
\end{gathered}
$$

We proceed as in the proof of Proposition 3.4. Magma [BCP97 produces the invariants $L_{1}, L_{2}$ and $L_{3}$ as generators of the algebra of invariants $K[M, N]^{G}$ for the fields $\mathbb{F}_{3}$ and $\mathbb{Q}$. Again, Molien's Formula [DK02, Theorem 3.2.2] extends the result to any field of characteristic different from 2 .

Remark 5.3. The following equalities hold: $\Delta(X)=L_{2}$ and $\Delta(Y)=2^{4} L_{2}^{4} L_{3}^{2}$.

Remark 5.4. If the curve $Y \in \mathcal{M}_{3, V}^{\text {hyp }}$ has a model as in (5.1) given by the parameters $(M, N)$, then there is always a pair in (5.5) such that the valuation of both terms is nonnegative so again the valuation of the invariants $L_{i}$ can be assumed to be non-negative. In this situation, the valuation of the three invariants $L_{i}$ cannot be simultaneously positive. In that case $N \equiv-10 \bmod \pi$ because of $\nu\left(L_{1}\right)>0, M^{2} \equiv 16 \bmod \pi$ because of $\nu\left(L_{3}\right)>0$, but then $L_{2} \equiv 64 \bmod \pi$ and $\nu\left(L_{2}\right)$ cannot be positive.

Proposition 5.5. Let $y^{2}=x^{8}+M x^{6}+N x^{4}+M x^{2}+1$ be a hyperelliptic curve in $\mathcal{M}_{3, V}^{\text {hyp }}$ with invariants $L_{1}, L_{2}, L_{3}$ defined as in Proposition 5.2. It has potentially good reduction if and only if $\nu\left(L_{1}^{2} / L_{2}\right) \geq 0$ and $\nu\left(L_{2}^{3} / L_{3}^{2}\right)=0$.

Proof. By Remark 5.4, we can assume $M, N, L_{i} \in \mathcal{O}$ and at least one of the $L_{i}$ having valuation zero. If $\nu\left(L_{1}^{2} / L_{2}\right) \geq 0$ and $\nu\left(L_{2}^{3} / L_{3}^{2}\right)=0$ hold then we have $\nu\left(L_{1}\right) \geq \nu\left(L_{2}\right) / 2=$ $\nu\left(L_{3}\right) / 3 \geq 0$. This gives us $\nu\left(L_{2}\right)=\nu\left(L_{3}\right)=0$ and $\nu(\Delta(Y))=\nu\left(2^{4} L_{2}^{4} L_{3}^{2}\right)=0$. Hence the curve has good reduction.

Conversely, assume the curve has potentially good reduction, then there exists a hyperelliptic curve model of $Y$ having good reduction. Because of Corollary 3.5 in [LLLR19] this model can be taken of the form $y^{2}=x^{8}+M x^{6}+N x^{4}+M x^{2}+1$ with $M, N \in \mathcal{O}$ and $\nu(\Delta(Y))=0$. Now, because of Remark 5.4 we can also assume $\nu\left(L_{1}\right), \nu\left(L_{2}\right), \nu\left(L_{3}\right) \geq 0$. So $\nu\left(L_{1}^{2} / L_{2}\right) \geq 0$ and $\nu\left(L_{2}^{3} / L_{3}^{2}\right)=0$.

5.2. The main theorem and its proof. We characterize the possible reduction types of a genus-3 hyperelliptic curve $Y$ in $\mathcal{M}_{3 V}^{\text {hyp }}$ in terms of the invariants $L_{1}, L_{2}$ and $L_{3}$ defined in Proposition 5.2. Because of Proposition 5.5, and once the invariants are normalized as in Remark 5.4, $Y$ has potentially good reduction if and only if $\nu(\Delta(Y))=0$. The theorem below describes the different types of bad reduction when $\nu(\Delta(Y))>0$.

Theorem 5.6. Let $Y$ be a hyperelliptic genus-3 curve defined by $Y: t^{2}=y^{4}+M y^{3} z+$ $N y^{2} z^{2}+M y z^{3}+z^{4}, x^{2}=y z \subseteq \mathbb{P}_{1,1,1,2}^{3}$. Let $X$ be the conic $Y /\left\langle\sigma_{1}, \sigma_{2}\right\rangle$ with $\sigma_{1}, \sigma_{2}$ given as in (5.2). Then if the valuation of $\Delta(Y)$ is positive, $Y$ has geometric bad reduction and one of the cases in Table 5.1 occurs. 


\begin{tabular}{|c|c|c|c|c|c|c|}
\hline & $\nu\left(L_{1}\right)$ & $\nu\left(L_{2}\right)$ & $\nu\left(L_{3}\right)$ & Other conditions & Decorated graphs & Stable curve \\
\hline (a) & & $=0$ & $>0$ & $\nu\left(L_{1}^{2}-4 L_{2}\right)=0$ & II.3 & Loop \\
\hline (b) & $=0$ & $=0$ & $>0$ & $\nu\left(L_{1}^{2}-4 L_{2}\right)>0$ & III.1 & DNA \\
\hline (c) & & $>0$ & $=0$ & & II.2 & DNA \\
\hline (d.i) & & & & $\nu\left(L_{2}\right)<\nu\left(L_{3}\right)$ & $\mathrm{IV}^{*} .2$ & DNA \\
\hline (d.ii) & & $>0$ & $>0$ & $\nu\left(L_{2}\right)>\nu\left(L_{3}\right)$ & IV.5 & Braid \\
\hline (d.iii) & & & & $\nu\left(L_{2}\right)=\nu\left(L_{3}\right)$ & II. 4 & Candy \\
\hline
\end{tabular}

TABLE 5.1. Cases of Theorem 5.6.

Proof. Recall $\Delta(Y)=2^{4} L_{3}^{2} \Delta(X)^{4}$ and $\Delta(X)=L_{2}$, and assume $\nu(\Delta(Y))>0$.

If the valuation $\nu\left(L_{2}\right)$ is zero, then $\nu\left(L_{3}\right)>0$ holds, i.e., at least one of the valuations $\nu(2 M+N+2), \nu(-2 M+N+2)$ is positive; and the conic $X$ has good reduction. If exactly one of them is positive, that is, $\nu(N+2)=0$, then the special fiber $\bar{X}$ of the stably marked model of $X$ is of type II.3. Otherwise, if both $\nu(2 M+N+2)$ and $\nu(-2 M+N+2)$ are positive, and hence $\nu(N+2)>0$ and $\nu\left(L_{1}\right)=0$, then the special fiber $\bar{X}$ of the stably marked model of $X$ is of type III.1. Statements (a) and (b) then follow from noticing that if $\nu\left(L_{1}\right)>0$, then we write

$$
2^{3} L_{3}-2^{2} L_{2} L_{1}+L_{1}^{3}=-(N+2)\left(L_{3}-2^{5} L_{1}\right)
$$

hence $\nu(N+2)>0$ if and only if $\nu\left(L_{1}^{2}-4 L_{2}\right)>0$.

Suppose now $\nu\left(L_{2}\right)>0$. Then by Remark 5.1, the conic $\bar{X}$ is a product of two lines. If $\nu\left(L_{3}\right)=0$, that is, we are in case (c), then the branch points $P_{1}$ and $P_{1}^{\prime}$ specialize to different lines by Proposition 4.9. (i), and the same holds for $P_{2}, P_{2}^{\prime}$ and $P_{3}, P_{3}^{\prime}$. So we obtain that $\bar{X}$ is of type II.2.

Finally we assume $\nu\left(L_{2}\right)>0, \nu\left(L_{3}\right)>0$. We write

$$
L_{2}=(M+4-2 \lambda)(M+4+2 \lambda)=(M-4-2 \mu)(M-4+2 \mu),
$$

where $\lambda$ is a root of $T^{2}-(2 M+N+2)$ with $\nu(\lambda)>0$ and $\mu$ is a root of $T^{2}-(-2 M+N+2)$ with $\nu(\mu)=0$.

Notice that we get $\nu(M+4)>0$ and $\nu(M-4)=0$ by (5.6). Then the conic $X$ reduces to a product of two lines and the branch points $P_{2}$ and $\overline{P_{2}^{\prime}}$ specialize to $(1: 4: 0)$, the intersection of the lines.

Consider the coordinate

$$
\xi=\frac{-(4+\lambda) u+v+w}{(4-\lambda) u-v+w}
$$

whhere $u, v, w$ are the coordinates of $X$ as in (5.3), and which satisfies $\xi\left(P_{1}\right)=\infty$, $\xi\left(P_{1}^{\prime}\right)=0, \xi\left(P_{2}^{\prime}\right)=1$ and $\xi\left(P_{2}\right)=(M+4-2 \lambda)(M+4+2 \lambda)^{-1}$. Depending on the value of $\overline{\xi\left(P_{2}\right)}$ we get different possibilities for the decorated graph. We have:

$$
\overline{\xi\left(P_{2}\right)}= \begin{cases}\infty & \text { iff } \nu(M+4+2 \lambda)>\nu(M+4-2 \lambda),(\text { type IV.5), } \\ 0 & \text { iff } \nu(M+4+2 \lambda)<\nu(M+4-2 \lambda),(\text { type IV.5), } \\ 1 & \text { iff } \nu(M+4+2 \lambda)=\nu(M+4-2 \lambda)=\nu(M+4)<\nu(\lambda),\left(\text { type IV }^{*} .2\right), \\ \neq 0, \infty, 1 & \text { iff } \nu(M+4+2 \lambda)=\nu(M+4-2 \lambda)=\nu(\lambda) \leq \nu(M+4), \text { (type III.4). }\end{cases}
$$

The different cases there can be rewritten as: 
(1) Case IV*.2 if and only if $2 \nu\left(M^{2}-16\right) \geq \nu\left(L_{2}\right)$ and $2 \nu\left(M^{2}-16\right)<\nu\left(L_{3}\right)$,

(2) Case IV.5 if and only if $2 \nu\left(M^{2}-16\right)<\nu\left(L_{2}\right)$, and

(3) Case III. 4 if and only if $2 \nu\left(M^{2}-16\right) \geq \max \left\{\nu\left(L_{2}\right), \nu\left(L_{3}\right)\right\}$;

and one can check that these conditions are equivalent to the ones in the statement.

Corollary 5.7. (i) The $j$-invariant of the genus-1 component of the special fiber in Theorem 5.6. (a) is $j=2^{4}\left(12 L_{2}+L_{1}^{2}\right)^{3} /\left(\left(4 L_{2}-L_{1}^{2}\right)^{2} L_{2}\right)$.

(ii) The j-invariants of the two genus-1 components of the special fiber in Theorem 5.6. (d.iii) are equal to 1728.

Proof. (i) In order to compute the $j$-invariant of the elliptic curve component $E$ of $\bar{Y}$ in case (a), we assume first that $\nu(-2 M+N+2)>0$. Then modulo $\pi$ the equation of $Y$ reduces to

$$
y^{2}=\left(x^{2}+1\right)^{2}\left(x^{4}+(M-2) x^{2}+1\right),
$$

so the elliptic curve we are looking for is $\left(\frac{y}{x^{2}+1}\right)^{2}=\left(x^{4}+(M-2) x^{2}+1\right)$ with

$$
j=\frac{2^{4}\left(12 L_{2}+L_{1}^{2}\right)^{3}}{\left(4 L_{2}-L_{1}^{2}\right)^{2} L_{2}} \quad \bmod \pi .
$$

(ii) In order to compute the $j$-invariants of the two elliptic curves in case (d.iii) we proceed as in Lemma 4.11.(iii). to get that the two elliptic curves are isomorphic between them and isomorphic to the intermediate elliptic curves $E_{1}=Y /\left\langle\sigma_{1}\right\rangle$ and $E_{2}=Y /\left\langle\sigma_{2}\right\rangle$ or $E_{3}=Y /\left\langle\sigma_{3}\right\rangle$ depending on $\pm 2 M+N+2$ having positive valuation. The elliptic curve $E_{1}$ is given by the equation:

$$
y^{2}=x^{4}+M x^{3}+N x^{2}+M x+1,
$$

and we have that $M= \pm 4+m \pi^{r}$ and $N=6+n \pi^{2 r}$, hence with $j$-invariant:

$$
j \equiv 1728 \bmod \pi \text {. }
$$

\section{REFERENCES}

[BBW17] M. Börner, I. I. Bouw, and S. Wewers. Picard curves with small conductor. In Algorithmic and experimental methods in algebra, geometry, and number theory, pages 97-122. Springer, Cham, 2017.

$\left[\mathrm{BCK}^{+} 20\right]$ I. I. Bouw, N. Coppola, P. Kılıçer, S. Kunzweiler, E. Lorenzo García, and A. Somoza. Invariants generate DO. magma package. github.com/NirvanaC93/Invariants-Special-Strata-Genus-3-Curves, 2020.

[BCP97] W. Bosma, J. Cannon, and C. Playoust. The Magma algebra system. I. The user language. J. Symbolic Comput., 24(3-4):235-265, 1997. Computational algebra and number theory (London, 1993).

[BKSW] I. I. Bouw, A. Koutsianas, J. Sijsling, and S. Wewers. Conductor and discrimant of Picard curves. To appear in J. London Math. Soc., http://front.math.ucdavis .edu/1902.09624.

[Bou98] I. I. Bouw. Tame covers of curves: p-ranks and fundamental groups. PhD thesis, Utrecht University, 1998.

[BW17] I. I. Bouw and S. Wewers. Computing $L$-functions and semistable reduction of superelliptic curves. Glasg. Math. J., 59(1):77-108, 2017.

[Cia99] E. Ciani. I varii tipi possibili di quartiche piane più volte omologico-armoniche. Palermo Rend., 13:347-373, 1899.

[DDMM19] T. Dokchitser, V. Dokchitser, C. Maistret, and A. Morgan. Semistable types of hyperelliptic curves. In Algebraic curves and their applications, volume 724 of Contemp. Math., pages 73-135. Amer. Math. Soc., Providence, RI, 2019.

[Dem12] M. Demazure. Résultant, discriminant. Enseign. Math. (2), 58(3-4):333-373, 2012. 
[Dix87] J. Dixmier. On the projective invariants of quartic plane curves. Adv. in Math., 64:279-304, 1987.

[DK02] H. Derksen and G. Kemper. Computational invariant theory. Invariant Theory and Algebraic Transformation Groups, I. Springer-Verlag, Berlin, 2002. Encyclopaedia of Mathematical Sciences, 130.

[DM69] P. Deligne and D. Mumford. The irreducibility of the space of curves of given genus. Inst. Hautes Études Sci. Publ. Math., (36):75-109, 1969.

[Hen76] P.-G. Henn. Die Automorphismengruppen der algebraischen Funktionenkörper vom Geschlecht 3. PhD thesis, Heidelberg, 1976.

[HLP00] E. W. Howe, F. Leprévost, and B. Poonen. Large torsion subgroups of split Jacobians of curves of genus two or three. Forum Math., 12(3):315-364, 2000.

[Liu93] Q. Liu. Courbes stables de genre 2 et leur schéma de modules. Math. Ann., 295(2):201-222, 1993.

[LLLR19] R. Lercier, Q. Liu, E. Lorenzo García, and C. Ritzenthaler. Reduction type of smooth quartics. To appear in Algebra $\&$ Number Theory, 2019. https://arxiv.org/abs/1803. 05816.

[LR08] G. Lachaud and C. Ritzenthaler. On some questions of Serre on abelian threefolds. In Algebraic geometry and its applications, volume 5 of Ser. Number Theory Appl., pages 88-115. World Sci. Publ., Hackensack, NJ, 2008.

[LR12] R. Lercier and C. Ritzenthaler. Hyperelliptic curves and their invariants: geometric, arithmetic and algorithmic aspects. J. Algebra, 372:595-636, 2012.

[LRRS14] R. Lercier, C. Ritzenthaler, F. Rovetta, and J. Sijsling. Parametrizing the moduli space of curves and applications to smooth plane quartics over finite fields. LMS J. Comput. Math., 17(suppl. A):128-147, 2014.

[LRS16] R. Lercier, C. Ritzenthaler, and J. Sijsling. quartic_reconstruction; a magma package for reconstructing plane quartics. https://github.com/JRSijsling/quartic_reconstruction, 2016.

[LRS18] R. Lercier, C. Ritzenthaler, and J. Sijsling. Reconstructing plane quartics from their invariants. Discrete $\mathcal{E}$ Computational Geometry, pages 1-41, 2018.

[Ohn07] T. Ohno. The graded ring of invariants of ternary quartics I, 2007. unpublished.

[RW06] M. Romagny and S. Wewers. Hurwitz spaces. In Groupes de Galois arithmétiques et différentiels, volume 13 of Sémin. Congr., pages 313-341. Soc. Math. France, Paris, 2006.

[Shi67] T. Shioda. On the graded ring of invariants of binary octavics. American J. of Math., 89(4):1022-1046, 1967.

[Sil09] J. H. Silverman. The arithmetic of elliptic curves, volume 106 of Graduate Texts in Mathematics. Springer, Dordrecht, second edition, 2009.

[Sut18] A. Sutherland. A database of nonhyperelliptic genus 3 curves over $\mathbb{Q}$. https://arxiv.org/ abs/1806.06289, 2018.

[Ver83] A. Vermeulen. Weierstrass points of weight two on curves of genus three. $\mathrm{PhD}$ thesis, University of Amsterdam, Amsterdam, 1983.

[Wew99] S. Wewers. Deformation of tame admissible covers of curves. In Aspects of Galois theory (Gainesville, FL, 1996), volume 256 of London Math. Soc. Lecture Note Ser., pages 239282. Cambridge Univ. Press, Cambridge, 1999.

Irene Bouw, Universität Ulm, Institut für Reine Mathematik, D-89081 Ulm, Germany. Email address: irene.bouw@uni-ulm.de

Nirvana Coppola, University of Bristol, School of Mathematics, BS8 1UG Bristol, United KINGDOM.

Email address: nc17051@bristol.ac.uk

Pınar Kılıçer, Bernoulli Institute for Mathematics, Computer Science and Artificial Intelligence, 9747 AG Groningen, Netherlands

Email address: p.kilicer@rug.nl

Sabrina Kunzweiler, Universität Ulm, Institut fÜr Reine Mathematik, D-89081 Ulm, GerMANY.

Email address: sabrina.kunzweiler@uni-ulm.de 
Elisa Lorenzo García, Univ Rennes, CNRS, IRMAR - UMR 6625, F-35000 Rennes, France. Email address: elisa.lorenzogarcia@univ-rennes1.fr

Anna Somoza, Univ Rennes, CNRS, IRMAR - UMR 6625, F-35000 Rennes, France.

Email address: anna.somoza@univ-rennes1.fr 
Appendix A. Admissible covers

\begin{tabular}{cccc}
\hline Stable curve & Decorated graph & Stable curve & Decorated graph \\
\hline Good & I & Winky Cat & III.6 \\
Candy & II.1 & Cave & III.7 \\
DNA & II.2 & Grl Pwr & IV.1 \\
Loop & II.3 & Garden & IV.2 \\
Lop & II.4 & Cat & IV.3 \\
DNA & III.1 & Braid & IV.4 \\
Looop & III.2 & Braid & IV.5 \\
Loop & III.3 & Braid & IV*.1 \\
Candy & III.4 & DNA & IV $^{*} .2$ \\
Tree & III.5 & Looop & IV*.3 $^{*}$
\end{tabular}

TABLE A.1. Correspondence between the decorated graphs in Figures (I)$\left(\overline{I V}{ }^{*}\right)$ and the stable curves in Figure A.2.

FigURE I. Stably marked curve with 6 marked points and one component.

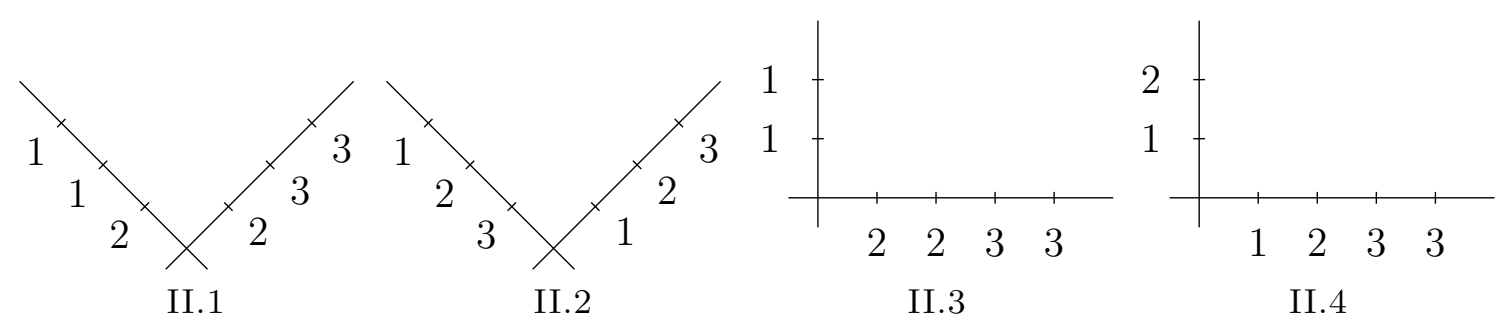

Figure II. Stably marked curves with 6 marked points and two components. 


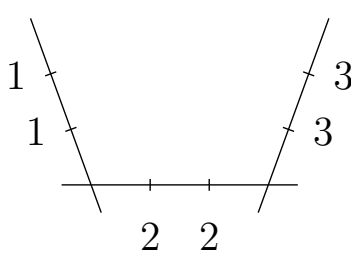

III.1

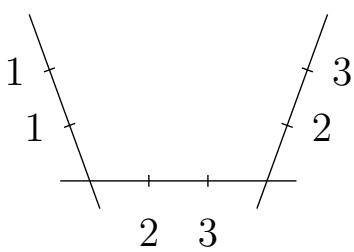

III. 2

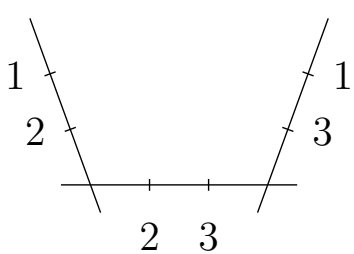

III.3

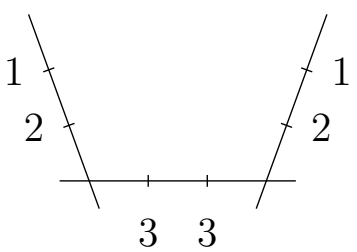

III. 4

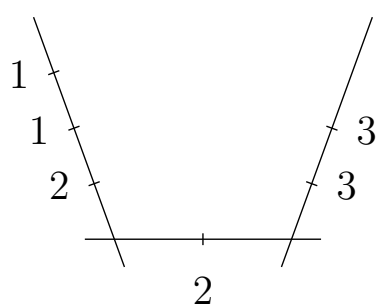

III.5

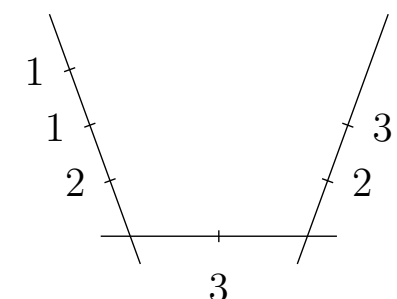

III.6

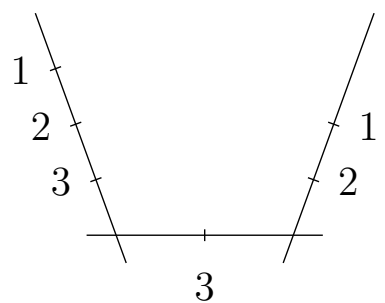

III. 7

FiguRE III. Stably marked curves with 6 marked points and three components.

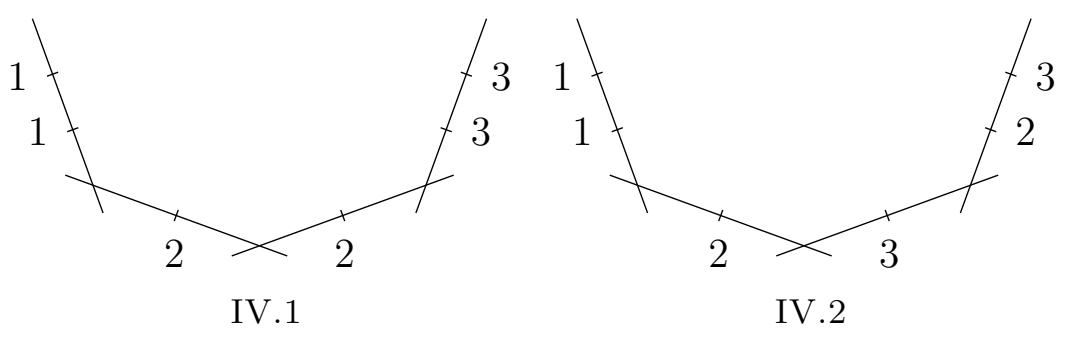

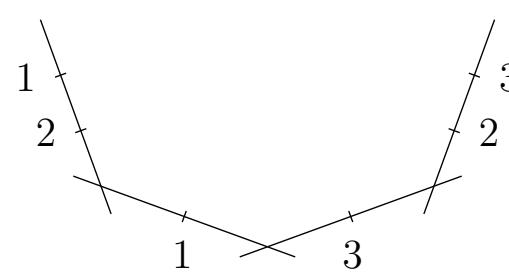

IV.3

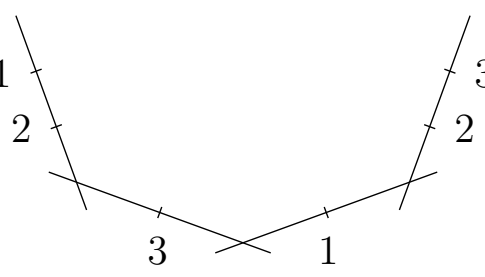

IV.4

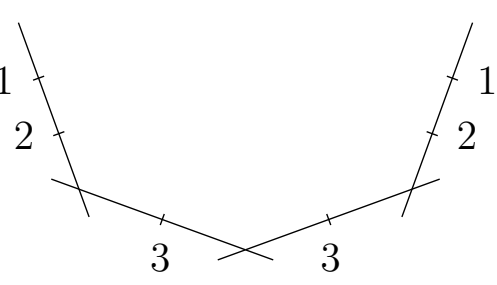

IV.5

Figure IV. Stably marked curves with 6 marked points and four components, all containing at least one marked point.

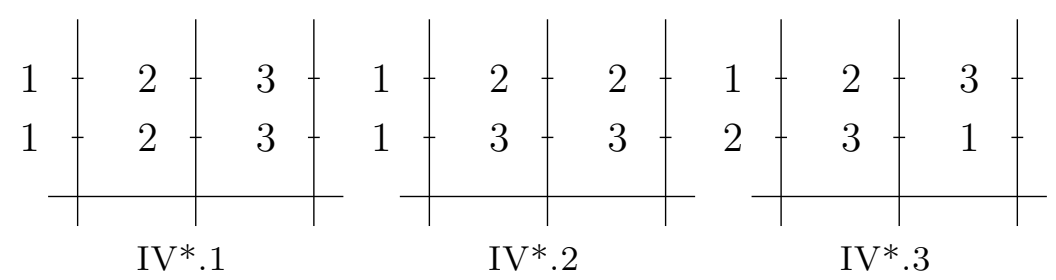

FiguRE IV*. Stably marked curves with 6 marked points and four components, one of which doesn't contain any marked point. 


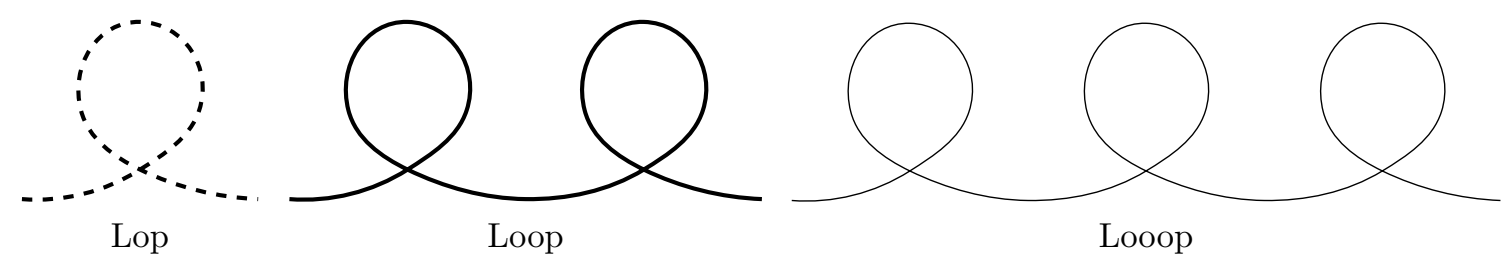

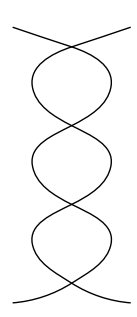

DNA

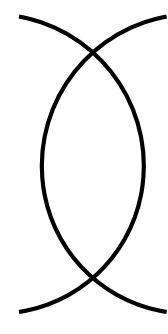

Candy

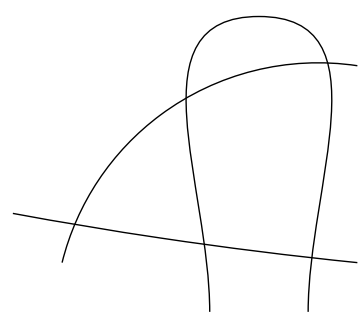

Cave

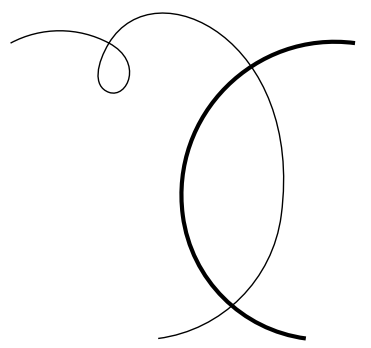

Winky cat
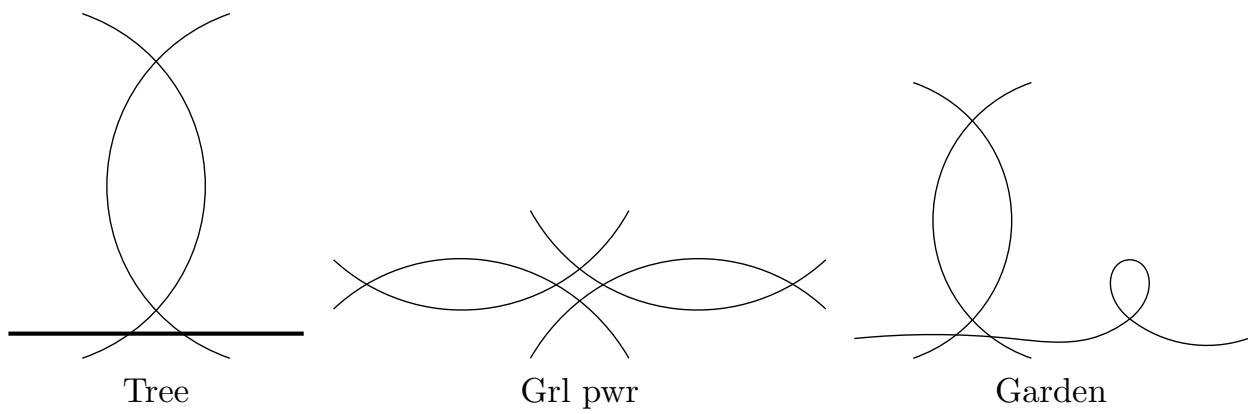

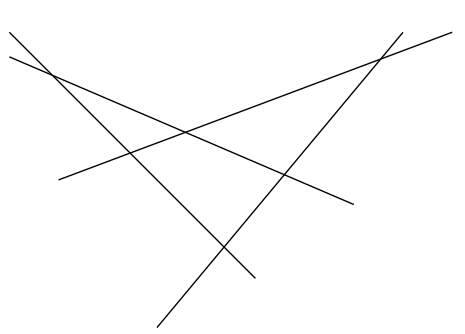

Braid

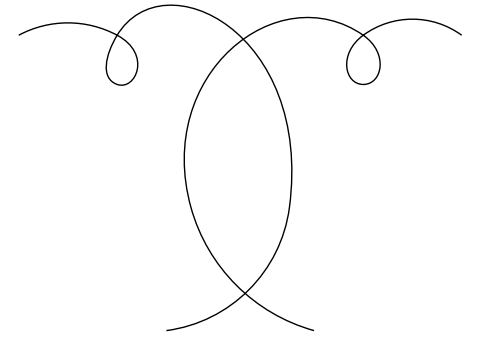

Cat

Figure A.2. Admissible covers. The genus-2 components correspond to the thick dashed lines, and the genus-1 components correspond to the thick solid lines. The remaining components have genus 0 . 